\title{
Neurotrophic factor GDNF regulates intestinal barrier function in inflammatory bowel disease
}

\author{
Michael Meir, ${ }^{1}$ Natalie Burkard, ${ }^{1}$ Hanna Ungewiß, ${ }^{2}$ Markus Diefenbacher, ${ }^{3}$ Sven Flemming, ${ }^{1}$ Felix Kannapin, ${ }^{1}$ \\ Christoph-Thomas Germer, ${ }^{1}$ Matthias Schweinlin, ${ }^{4}$ Marco Metzger, ${ }^{4,5}$ Jens Waschke, ${ }^{2}$ and Nicolas Schlegel ${ }^{1}$ \\ 'Department of Ceneral, Visceral, Vascular and Pediatric Surgery, University Hospital Wuerzburg, Wuerzburg, Germany. ${ }^{2}$ Institute of Anatomy and Cell Biology, Ludwig-Maximilians-Universität München, \\ Munich, Germany. ${ }^{3}$ Department of Biochemistry and Molecular Biochemistry, University of Wuerzburg, Wuerzburg, Germany. ${ }^{4}$ Department for Tissue Engineering and Regenerative Medicine, University \\ Hospital Wuerzburg, Wuerzburg, Germany. ${ }^{5}$ Fraunhofer ISC, Translational Centre Regenerative Medicine TLC-RT, Wuerzburg, Germany.
}

\begin{abstract}
Impaired intestinal epithelial barrier (IEB) function with loss of desmosomal junctional protein desmoglein 2 (DSC2) is a hallmark in the pathogenesis of inflammatory bowel disease (IBD). While previous studies have reported that glial cell line-derived neurotrophic factor (GDNF) promotes IEB function, the mechanisms are poorly understood. We hypothesized that CDNF is involved in the loss of DSC2, resulting in impaired IEB function as seen in IBD. In the inflamed intestine of patients with IBD, there was a decrease in GDNF concentrations accompanied by a loss of DSC2, changes of the intermediate filament system, and increased phosphorylation of p38 MAPK and cytokeratins. DSC2-deficient and RET-deficient Caco2 cells revealed that GDNF specifically recruits DSC2 to the cell borders, resulting in increased DSC2-mediated intercellular adhesion via the RET receptor. Challenge of Caco2 cells and enteroids with proinflammatory cytokines as well as dextran sulfate sodium-induced (DSS-induced) colitis in C57BI/6 mice led to impaired IEB function with reduced DSC2 mediated by p38 MAPK-dependent phosphorylation of cytokeratins. GDNF blocked all inflammation-induced changes in the IEB. GDNF attenuates inflammation-induced impairment of IEB function caused by the loss of DSC2 through p38 MAPK-dependent phosphorylation of cytokeratin. The reduced GDNF in patients with IBD indicates a disease-relevant contribution to the development of IEB dysfunction.
\end{abstract}

\section{Introduction}

Inflammatory bowel disease (IBD), encompassing Crohn's disease (CD) and ulcerative colitis (UC), is caused by a complex interplay between environmental factors, the composition of gut microbiota, and an inappropriate immune response in genetically predisposed individuals $(1,2)$. Recent evidence indicates that dysregulation of the intestinal epithelial barrier (IEB) plays a major role in the development, perpetuation, and severity of IBD (3) - especially under inflammatory conditions (4). Therefore, investigations into the underlying mechanisms behind inflammation-induced IEB breakdown in IBD are needed to identify novel therapeutic approaches.

Under basal conditions, the intestinal mucosa is lined by a single layer of polarized epithelial cells (enterocytes) that are sealed and held together by different junctional proteins such as tight junctions, adherens junctions, and desmosomes $(5,6)$. It has been shown previously that not only profound changes in tight junction integrity but also the loss of the desmosomal cadherin desmoglein 2 (DSG2) plays a critical role in the pathogenesis of intestinal barrier dysfunction in patients with CD (7). This is corroborated by recent findings from enterocyte-specific DSG2-deficient mice, which have impaired IEB both under basal and inflammatory conditions (8).

Conflict of interest: The authors have declared that no conflict of interest exists. Copyright: (5) 2019, American Society for Clinical Investigation.

Submitted: February 13, 2018; Accepted: May 3, 2019; Published: June 17, 2019.

Reference information: / Clin Invest. 2019;129(7):2824-2840.

https://doi.org/10.1172/JCl120261.
Desmosomes in the intestinal epithelium consist of the cadherin-type adhesion molecules DSG2 and desmocollin2 (DSC2) which are tethered to the intermediate filament cytoskeleton through specific desmosomal plaque proteins. Consequently, desmosomal function is dependent on a dynamic process in the intermediate filament system which is regulated by p38 mitogen-activated protein kinase (p38 MAPK) (9). The proinflammatory cytokine tumor necrosis factor $\alpha$ (TNF- $\alpha$ ) is one of the major cytokines in the pathogenesis of IBD (10) and has been shown to increase intestinal epithelial permeability by p38 MAPK-dependent loss of DSG2-mediated adhesion (7).

The enteric nervous system (ENS) and factors secreted by enteric glial cells are critically involved in the regulation of IEB function, and thus may be an unappreciated pathogenicity factor in the development of IBD (11). Additionally, the number of enteric glial cells is reduced in intestinal tissue samples of IBD patients, and toxic or autoimmune ablation of enteric glial cells leads to impaired IEB function in experimental studies in vivo $(12,13)$. However, this view was previously challenged since ablation of enteric glial cells in mice did not lead to alterations of IEB function (14). Nonetheless, a potential key player that was initially thought to be mainly secreted by enteric glial cells is glial cell line-derived neurotrophic factor (GDNF). Recent studies indicate that GDNF also derives from smooth muscle cells and enterocytes in significant amounts $(15,16)$. Loss of GDNF in experimental models leads to morphological and functional abnormalities of IEB similar to those seen in patients with IBD $(17,18)$. Furthermore, GDNF shows antiinflammatory effects in a murine model of colitis (19) and exerts direct effects on entero- 
A

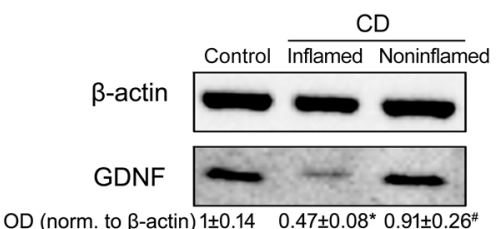

B

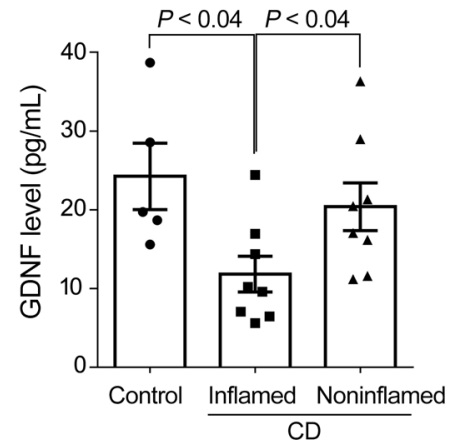

E
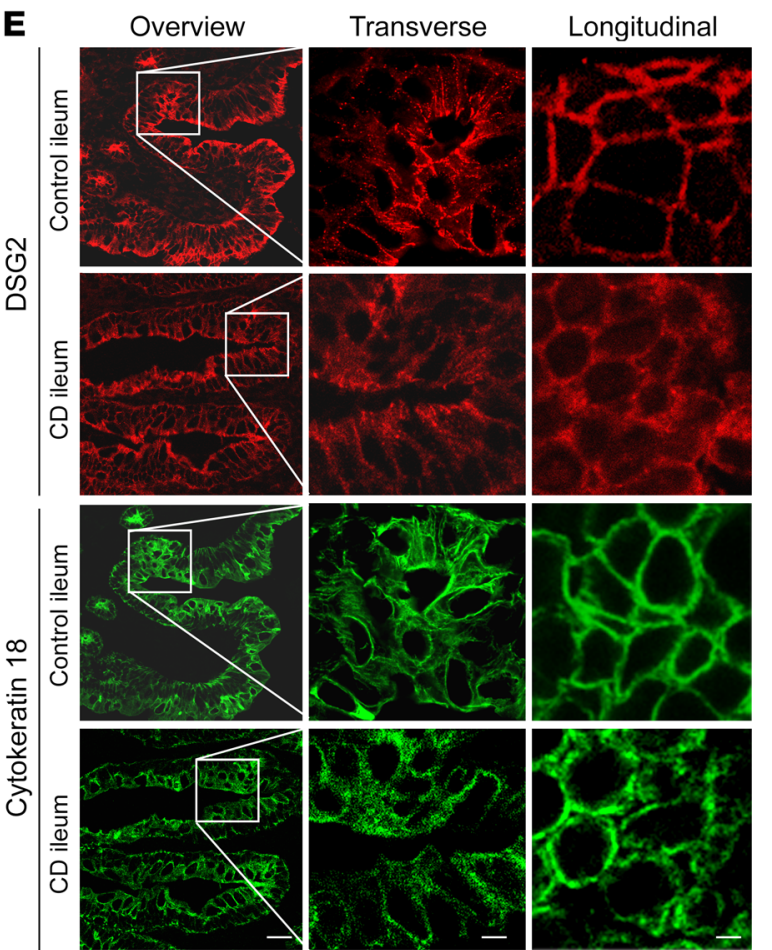

$\mathbf{F}$

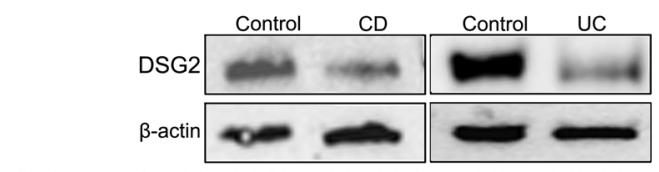

$\mathrm{OD}$ (norm. to $\beta$-actin) $1 \pm 0.31 \quad 0.21 \pm 0.05^{\star} \quad 1 \pm 0.12 \quad 0.42 \pm 0.08$

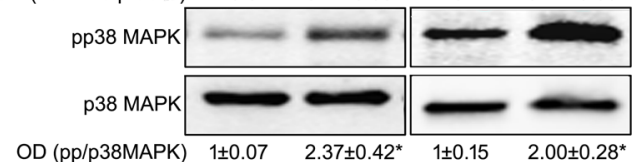

C

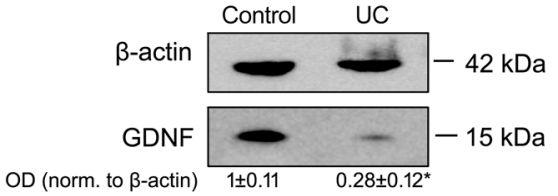

D
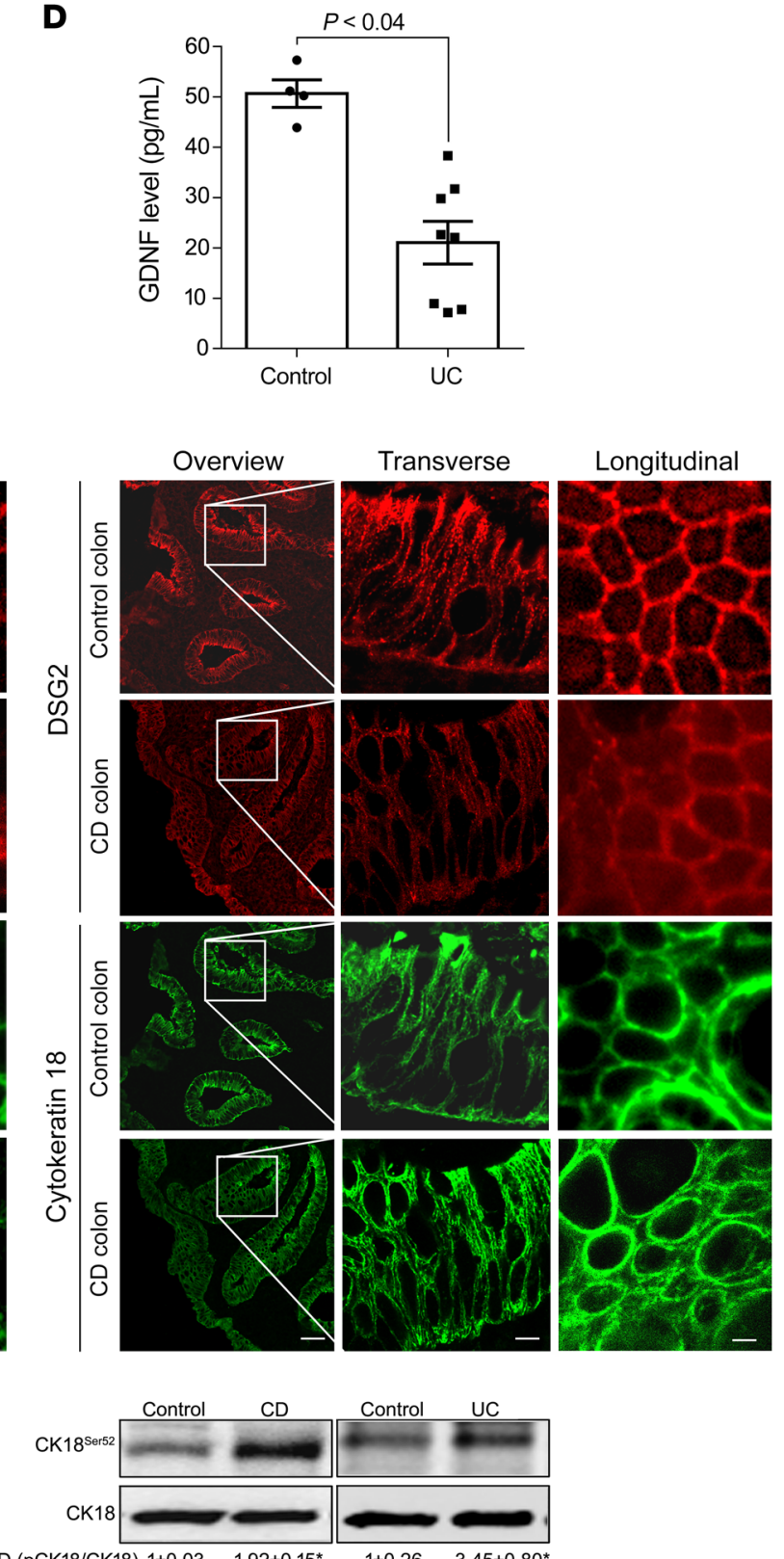

OD (pCK18/CK18) $1 \pm 0.03 \quad 1.92 \pm 0.15^{*} \quad 1 \pm 0.26 \quad 3.45 \pm 0.80^{*}$

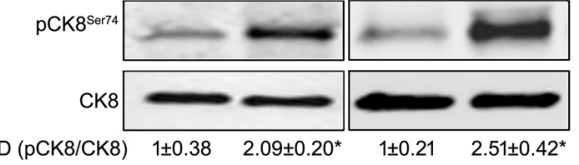

Figure 1. Reduction of GDNF correlated with loss of DSG2 in patients with IBD. Western blot analyses (A and C) and GDNF ELISA (B and D) from resection specimens of patients with CD and UC showed significantly reduced levels of GDNF (Western blot, $n=9$; ELISA, $n=5$ control; CD or UC, $n=8$ ). Kruskal-Wallis test (ANOVA) was carried out for CD blots and ELISAs followed by a Mann-Whitney U test for UC blots or Dunn's multiple comparison test for ELISAs. (E) Immunostaining was performed for DSG2 or cytokeratin 18 from resection specimens from the terminal ileum of patients with CD or from the colon of patients with CU ( $n=9$ for each condition). Scale bars: $50 \mu \mathrm{m}$ for the overview panels; $10 \mu \mathrm{m}$ for the transverse/longitudinal panels. (F) Western blot analyses of control $(n=6), C D$, and UC samples $(n=9)$ of DSC2, p38 MAPK, cytokeratin 18, and cytokeratin 8 were performed. Kruskal-Wallis tests (ANOVA) were carried out. OD values normalized to $\beta$-actin or to total p38 MAPK, cytokeratin 18 , or cytokeratin 8 are indicated below the Western blots. ${ }^{*} P<0.05$ compared with control, ${ }^{\#} P<0.05$ compared with uninflamed tissue. 
Table 1. Patient characteristics

\begin{tabular}{|c|c|c|c|c|c|}
\hline Identification & Sex & Age & Medication & Histology & Location \\
\hline Control 1 & $\mathrm{~F}$ & 61 & None & NET & Ileum \\
\hline Control 2 & M & 66 & None & Colon asc. carcinoma & Ileum \\
\hline Control 3 & M & 60 & None & Coecum adenoma & Ileum \\
\hline Control 4 & $\mathrm{~F}$ & 76 & None & Colon asc. carcinoma & Ileum \\
\hline Control 5 & M & 49 & None & Colon asc. adenoma & Ileum \\
\hline Control 6 & M & 52 & None & Sigma carcinoma & Colon desc. \\
\hline Control 7 & $\mathrm{~F}$ & 70 & None & Colon asc. carcinoma & Colon trans. \\
\hline Control 8 & $\mathrm{~F}$ & 68 & None & Colon asc. carcinoma & Colon trans. \\
\hline Control 9 & M & 67 & None & Rectum carcinoma & Colon sig. \\
\hline Control 10 & M & 55 & None & Colon asc. carcinoma & Colon trans. \\
\hline Patient 1 & $\mathrm{~F}$ & 25 & AZT & Active Crohn's disease, stenosis & Ileum \\
\hline Patient 2 & M & 26 & Adalimumab & Active Crohn's disease & Ileum \\
\hline Patient 3 & M & 32 & Infliximab, prednisolone & Active Crohn's disease, stenosis & lleum \\
\hline Patient 4 & M & 29 & AZT & Active Crohn's disease & lleum \\
\hline Patient 5 & $\mathrm{~F}$ & 24 & Infliximab, mesalazine, prednisolone & Active Crohn's disease & Jejunum \\
\hline Patient 6 & $\mathrm{~F}$ & 41 & Budesonide, infliximab, AZT & Active Crohn's disease, retroperitoneal fistula & lleum \\
\hline Patient 7 & M & 50 & MTX & Active Crohn's disease, stenosis & Ileum \\
\hline Patient 8 & M & 46 & Budesonide & Active Crohn's disease & lleum \\
\hline Patient 9 & $\mathrm{~F}$ & 34 & Prednisolone & Active Crohn's disease & Ileum \\
\hline Patient 10 & M & 65 & Mesalazine & Colon asc. carcinoma, active ulcerative colitis & Colon desc. \\
\hline Patient 11 & M & 45 & Adalimumab & Active ulcerative colitis & Colon desc. \\
\hline Patient 12 & M & 21 & Prednisolone, infliximab & Active ulcerative colitis & Rectum \\
\hline Patient 13 & $\mathrm{~F}$ & 48 & Golimumab & Active ulcerative colitis & Colon sig. \\
\hline Patient 14 & M & 63 & None & Colon desc. carcinoma, active ulcerative colitis & Colon trans. \\
\hline Patient 15 & M & 55 & Budesonide, mesalazine & Colon asc. carcinoma, active ulcerative colitis & Rectum \\
\hline Patient 16 & M & 41 & Vedolizumab & Active ulcerative colitis & Colon sig. \\
\hline Patient 17 & M & 53 & Infliximab, prednisolone & Active ulcerative colitis & Colon sig. \\
\hline Patient 18 & $\mathrm{~F}$ & 72 & Prednisolone, mesalazine & Rectum carcinoma, active ulcerative pancolitis & Colon trans. \\
\hline
\end{tabular}

Asc., ascendens; trans., transversum; desc., descendens; sig., sigmoideum.

cytes in vitro, leading to the maturation of tight junctions in the IEB by largely unknown mechanisms (16).

Based on this, we tested the hypothesis that GDNF critically regulates DSG2-dependent integrity of the intestinal barrier in inflammation and is thereby involved in the pathogenesis of intestinal barrier dysfunction in IBD.

\section{Results}

In inflammation, reduced GDNF in the terminal ileum and colon of patients with IBD is associated with loss of barrier function. Full wall tissue specimens from patients with CD and UC were compared to detect changes of intestinal GDNF concentrations in IBD. While GDNF concentrations were comparable in whole intestinal tissue lysates of healthy patients and in noninflamed intestinal tissue of patients suffering from $C D, G D N F$ was reduced in inflamed parts of the same CD tissue samples as revealed by Western blot analyses and ELISA-based measurements of GDNF concentrations (Figure 1, A and B). Similar results were evident in UC samples (Figure 1, C and D).

As shown by immunofluorescence staining, the loss of GDNF in CD and UC was paralleled by changes of the desmosomal adhesion protein DSG2 and the intermediate filament system such as cytokeratin 18 (CK18). Under basal (noninflamed) conditions, DSG2 was regularly distributed along the cell borders and CK18 was well organized (Figure 1E). In contrast, DSG2 was lost at the cell borders and the intermediate filament system was completely deranged in inflamed tissue of IBD patients (Figure 1E).

Western blot analyses of the human IBD samples showed a significant reduction of DSG2 (Figure 1F, Supplemental Figure 1A, and Supplemental Figure 2A; supplemental material available online with this article; https://doi.org/10.1172/JCI120261DS1). Since DSG2 is known to be regulated by p38 MAPK (20) and we observed alterations of cytokeratins in immunostaining, we tested whether phosphorylation of these proteins was altered in IBD. In CD and in UC samples, phosphorylation of p38 MAPK as well as phosphorylation of cytokeratins 18 and 8 were increased in Western blot analyses (Figure 1F, Supplemental Figure 1, B-D, Supplemental Figure 2, B-D, and Table 1). Western blotting of E-cadherin and claudin 1 served to exclude that the mucosa was lost in the tissue specimens from CD and UC patients (Supplemental Figure 1, $\mathrm{E}$ and $\mathrm{F}$, and Supplemental Figure 2, E and F).

GDNF effects on IEB are mediated via DSG2. These observations in patients led to the hypothesis that GDNF might be critically involved in the regulation of DSG2 and thereby contribute to loss of IEB function in IBD. As shown in our previous study (16), the presence of GDNF receptors RET, GFR $\alpha 1-3$ in Caco 2 cells, enteroids, and mouse and human tissue samples was confirmed by Western blotting (Supplemental Figure 6C). First, the effects of GDNF on DSG2 were evaluated in Caco2 
A
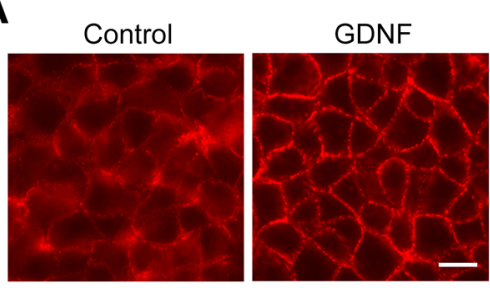

D

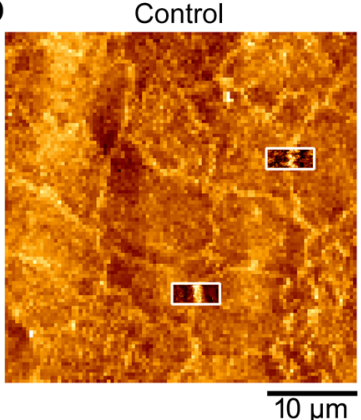

$\mathbf{F}$

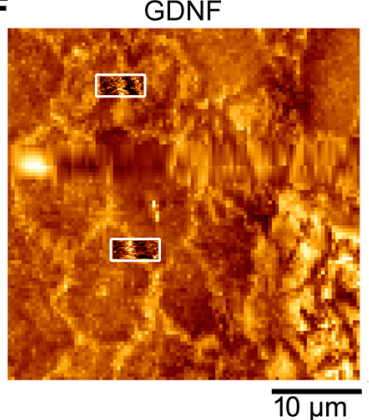

H
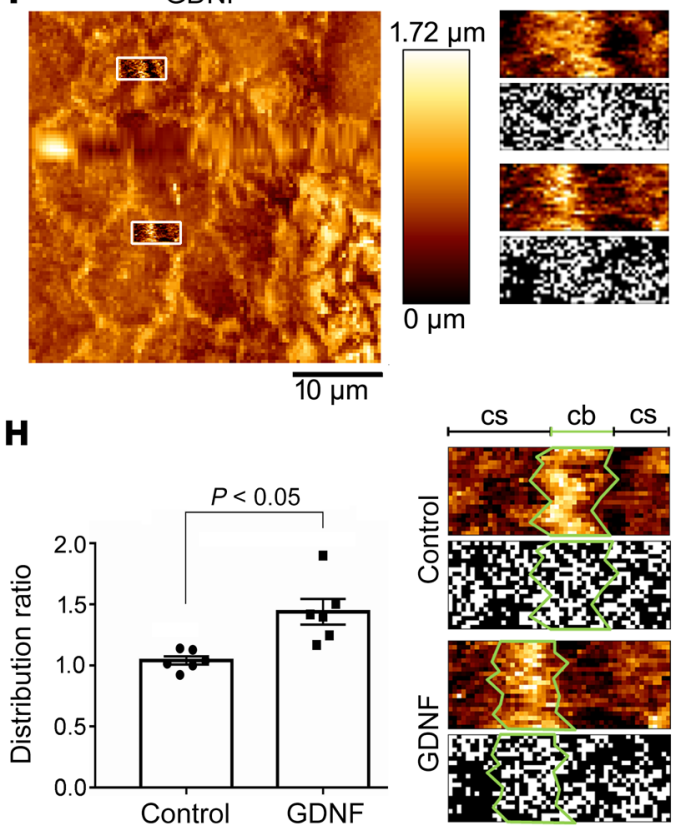

$0 \mu \mathrm{m}$
B

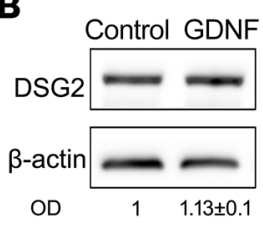

C

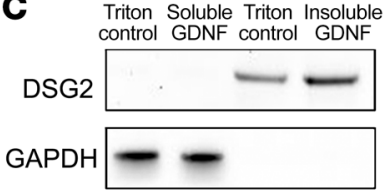

G
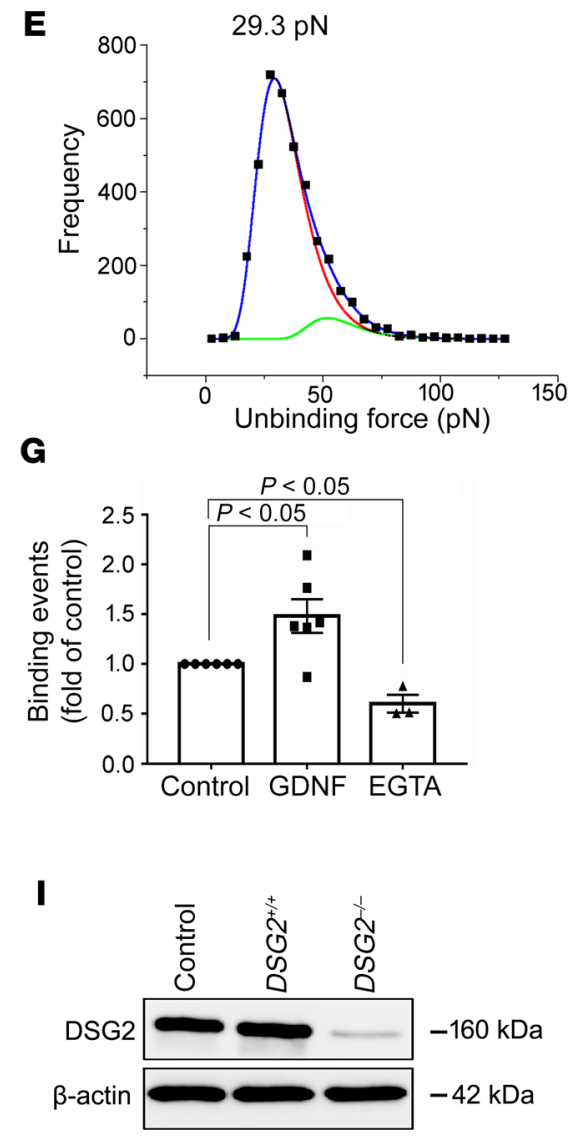

$O D$ (ß-Actin cor.) $1 \quad 1.10 \pm 0.9 \quad 0.09 \pm 0.01$

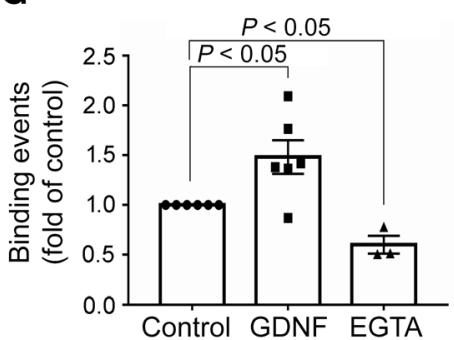

I
Figure 2. GDNF stabilizes the intestinal barrier via DSG2. (A)

Immunostaining of Caco2 monolayers at confluency for DSG2 and after application of $100 \mathrm{ng} / \mathrm{ml}$ GDNF for 24 hours; $n=10$. Scale bar, $20 \mu \mathrm{m}$. (B) Western blot for DSG2 after application of GDNF ( $n=5$; unpaired $t$ test). (C) DSG2 was augmented in the triton-insoluble fraction after GDNF application in triton extraction experiments $(n=$ 7; Kruskal-Wallis test, ANOVA). (D) In AFM measurements, living Caco2 cell topography images were created for selection of specific areas at cell borders (left panel). These areas (white boxes) were inserted as an overlay into the image to exemplify where measurements were carried out. Force measurements with a DSC2-coated AFM cantilever revealed binding events on the surface of $\mathrm{Caco} 2$ cells with each white dot representing 1 binding event (right panel). (E) Analysis of measured DSC2-specific unbinding forces resulted in a distribution peak of $29.3 \mathrm{pN}$. (F) Cell topography of Caco2 cells was imaged after incubation with GDNF (left panel). Areas surrounded by white boxes were inserted as an overlay into the image to exemplify where measurements were carried out. Force maps of DSG2 adhesion measurements display more binding events along the cell border (right panel) after application of GDNF. (C) Quantification of measured binding events showed increased binding frequency after application of GDNF and decreased binding frequency after EGTA-mediated $\mathrm{Ca}^{2+}$ depletion ( $n=6$ for GDNF, $n=3$ for EGTA; Kruskal-Wallis test, ANOVA) (H) Distribution ratio of DSC2-specific binding events was calculated as the quotient of measured binding events along the cell border (cb) and measured binding events on the surrounding cell surface (cs) ( $n=6$, paired $t$ test). (I) Western blot for DSG2 to confirm the effective knock out of DSG2 in the Caco2 DSG2-/- cell line ( $n=9$; KruskalWallis test, ANOVA). (J and K) TER measurements of Caco2 $\mathrm{DSC2}^{+/+}$cells transfected with nonsense plasmids (J) and Caco2 DSG2 $2^{-1-}$ following application of $100 \mathrm{ng} / \mathrm{ml}$ GDNF are shown $(n=$ 6 experiments for each condition). ${ }^{*} P$ $<0.05$; unpaired $t$ tests for each time point; OD values normalized to $\beta$-actin. monolayers. Immunostaining showed that application of 100 $\mathrm{ng} / \mathrm{ml}$ recombinant GDNF to confluent monolayers resulted in augmented staining patterns of DSG2 at the cell border (Figure $2 \mathrm{~A})$. While GDNF application did not increase total protein levels of DSG2 (Figure 2B), triton extraction assays showed DSG2 to be increased in the insoluble fraction, which is considered to contain cytoskeleton-bound and therefore membrane-associated proteins following GDNF treatment (Figure 2C). This indicated that GDNF recruits DSG2 to the cell border and thereby increases barrier formation. 
To explore the functional effect of GDNF on DSG2 binding properties and distribution on the cell surface, atomic force microscopy (AFM) was applied on living Caco2 cells (21). Imaging of the cell surface topography revealed a microvillus-covered surface typical for polarized Caco2 cells and elevated cell-cell borders, which allowed specific measurements at these areas (Figure 2D). To examine DSG2 binding properties, adhesion measurements with a DSG2-coated tip of a flexible AFM cantilever on living cells were performed. Two cell-cell border-containing areas were selected for each condition and 1000 force-distance curves were recorded for each area. To ensure comparability among different conditions, measurements under control conditions and on cells incubated with GDNF or EGTA were always performed with the same AFM cantilever. Under control conditions, binding events were detected with an unbinding force of around $29 \mathrm{pN}$ as revealed by peakfit analysis (Figure 2E), which resembles the values of previously measured DSG2-specific unbinding forces on the surface of living enterocytes (20). Application of GDNF increased the amount of measured binding events by approximately 50\% (Figure 2, F and G). EGTA was applied to demonstrate specificity of measured binding events as $\mathrm{Ca}^{2+}$ depletion disrupts $\mathrm{Ca}^{2+-}$-dependent cadherin binding. After 30 minutes of EGTA-mediated $\mathrm{Ca}^{2+}$ depletion, binding frequency was reduced by around $40 \%$ compared with control conditions (Figure $2 \mathrm{G}$ ). Since measured binding events after incubation with GDNF appeared to be more prominent at cell-cell borders (Figure 2F), it was examined next whether the localization of DSG2-specific binding events was altered compared with control conditions. Therefore, a distribution ratio defined as the percentage of measured binding events along the cell borders (cb) versus measured binding events on the cell surface (cs) was calculated (Figure $2 \mathrm{H}$ ). Indeed, the distribution ratio was increased after incubation with GDNF. Together, these data indicate that application of GDNF results in a redistribution of existing DSG2-specific interactions or promotes the emergence of new DSG2-specific interactions along the cell border.

To verify that the effects of GDNF on the IEB were specific for DSG2, a DSG2-deficient Caco2 (DSG2-/) cell line was generated using the CRISPR/CAS9 technique (Figure 2I). In Caco2 cells transfected with nonsense vectors, application of $100 \mathrm{ng} /$ $\mathrm{ml}$ recombinant GDNF led to increased transepithelial electrical resistance (TER) values of 1.5 -fold compared with untreated controls (Figure 2J). In DSG2/- Caco2 cells, application of GDNF did not show any effect on TER (Figure $2 \mathrm{~K}$ ), which confirmed that GDNF effects were dependent on DSG2.

$T N F-\alpha$-induced cytokeratin retraction and loss of DSG2 are blocked by GDNF. TNF- $\alpha$ was used to mimic inflammation-induced alterations in differentiated $\mathrm{Caco} 2$ monolayers because it is a key mediator that induces increased intestinal permeability in IBD. Application of TNF induced a significant reduction of TER to $0.73 \pm 0.05$-fold of baseline values after 8 hours (Figure $3 \mathrm{~A})$. Combined treatment of monolayers with TNF- $\alpha$ and GDNF completely blocked TNF-induced loss of TER. Similarly, measurements of $4 \mathrm{kDa}$ FITC dextran flux across Caco 2 monolayers revealed augmented epithelial permeability following incubation with TNF- $\alpha$ in both Caco2 $\mathrm{DSG}^{+/+}$and DSG2-deficient Caco 2 cells (Figure 3, B and C). Application of GDNF blocked this
TNF- $\alpha$-induced increase of epithelial permeability in $D S G 2^{+/+}$ cells but not in DSG2-deficient cells. Importantly, permeability measurements across WT and DSG2-deficient monolayers using $70 \mathrm{kDa}$ FITC-dextran was not changed following incubation with similar concentrations of TNF- $\alpha$ or TNF- $\alpha+$ GDNF (Supplemental Figure 3C). In line with this, gross cell damage by cell death/apoptosis under our experimental conditions was excluded as revealed by cell viability/apoptosis assays and activated caspase-3 Western blots (Supplemental Figure 4, A-E).

Following application of TNF- $\alpha$, DSG2 was reduced in triton extraction assays in the insoluble fraction i.e. in the cytoskeleton-bound fraction compared with controls. Combined treatment of cells with TNF- $\alpha$ and GDNF inhibited loss of DSG2 in the triton-insoluble fraction (Supplemental Figure 3, A and B). This observation was confirmed by immunostaining as DSG2 was regularly distributed at the cell border in differentiated Caco 2 cells (Figure 3D) under control conditions and was reduced after application of TNF- $\alpha$ (Figure 3D). The loss of DSG2 from the cell border was attenuated by simultaneous application of TNF- $\alpha$ and GDNF in Caco2 monolayers (Figure 3D). Intermediate filaments visualized by cytokeratin 18 immunostaining were present as a keratin ring in the cell periphery under control conditions (Figure 3D). Application of TNF- $\alpha$ led to a retraction of the intermediate filament ring (Figure 3D) which was diminished after combined treatment of cells with TNF and GDNF (Figure 3D). The visual impression of changes within the intermediate filament system were quantified and thereby confirmed by measurements of fluorescence intensity at the cell border and distance of retraction of the keratin ring from the cell border (Supplemental Figure 3, D and E).

In human intestinal enteroids, comparable observations were made for DSG2 and cytokeratin 18: While DSG2 was regularly distributed at the cell border under control conditions (Figure 4A), application of TNF- $\alpha$ led to decreased DSG2 staining (Figure 4A). Treatment of enteroids with TNF- $\alpha$ and GDNF blocked loss of DSG2 at the cell border (Figure 4A). The keratin ring (Figure 4A) was deranged after TNF- $\alpha$ application (Figure $4 \mathrm{~A}$ ) which was not observed following TNF- $\alpha$ and GDNF treatment (Figure 4A).

In dispase-based enterocyte dissociation assays, incubation of differentiated Caco2 (Caco DSG2 $2^{+/+}$) cells with TNF- $\alpha$ led to an increased number of cell fragments which was blocked by simultaneous application of TNF- $\alpha$ and GDNF (Figure 3F). In Caco2 DSG2 $2^{-/}$cells, we found increased cell dissociation compared with Caco $2 \mathrm{DSG}^{+/+}$cells under basal conditions. Cell dissociation was further raised after application of TNF- $\alpha$. However, in the absence of DSG2, increased cell dissociation was not blocked by application of GDNF (Figure 3G), confirming the specificity of GDNF effects on DSG2-mediated adhesion. Application of GDNF alone had no effect on basal cell dissociation in both cell lines.

Inflammation-induced effects on DSG2 are mediated by activation of p38 MAPK and phosphorylation of cytokeratin 18. Given the alternating activation or inactivation of p38 MAPK by TNF- $\alpha$ and GDNF, we hypothesized that epithelial barrier protection by GDNF is mediated by affecting p38 MAPK followed by modulation of the intermediate filament system. Accordingly, phosphorylation of p38 MAPK was increased following incubation with TNF- $\alpha$ in Caco 2 cells and in human enteroids (Figure 3E and Fig- 

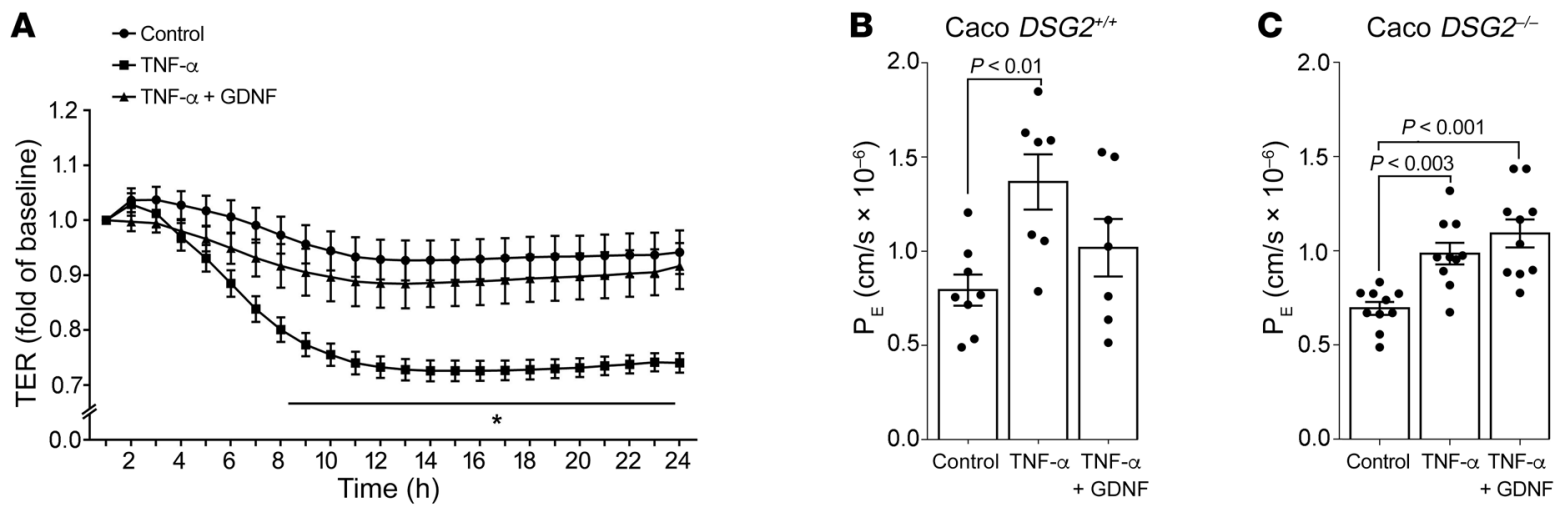

D
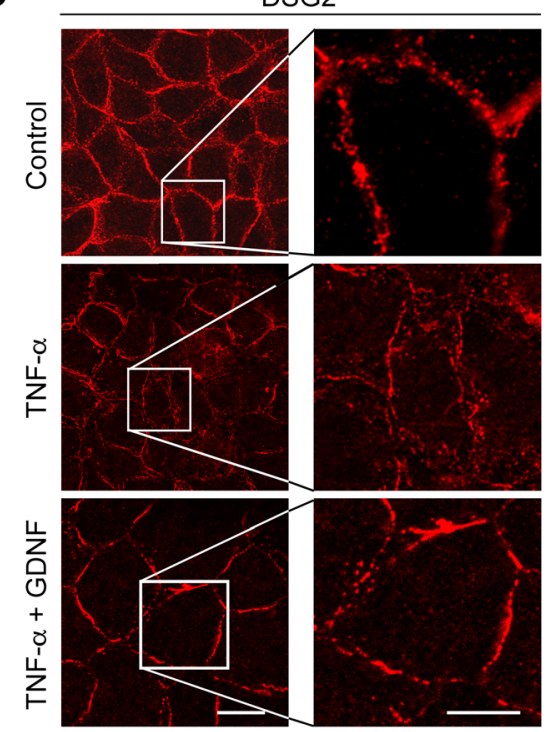

E
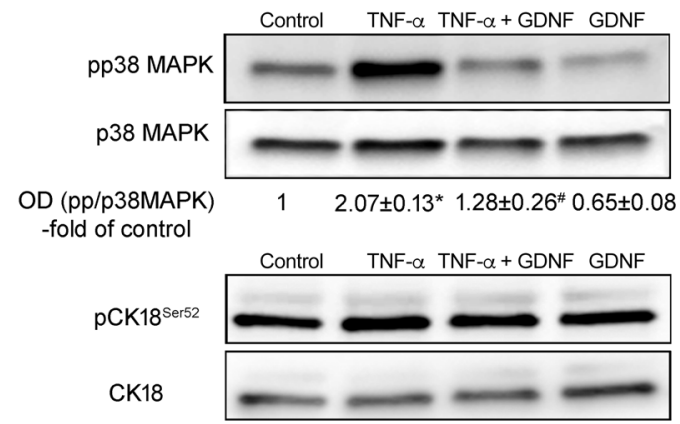

OD $\left(\right.$ pCK18/CK18) $1 \quad 1.44 \pm 0.16^{*} \quad 0.89 \pm 0.10^{*} 0.82 \pm 0.11$ -fold of control

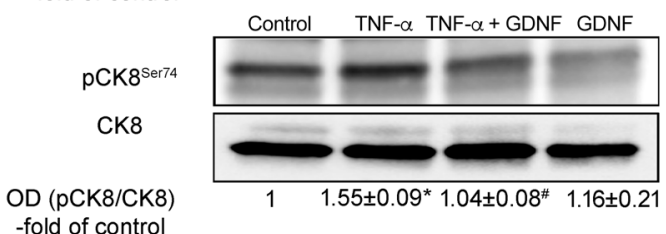

Cytokeratin 18

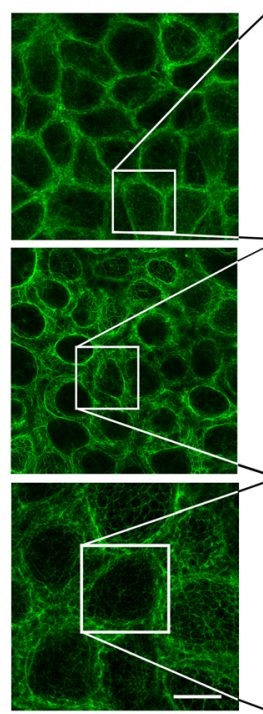

Merge

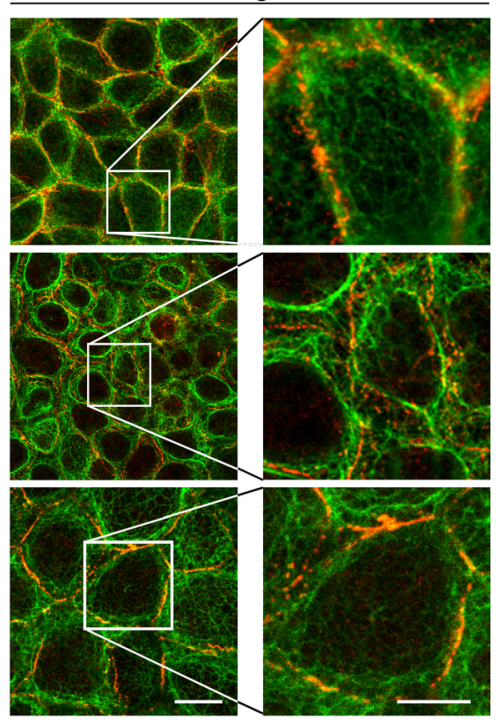

$\mathbf{F}$

Caco DSG2 $2^{+++}$

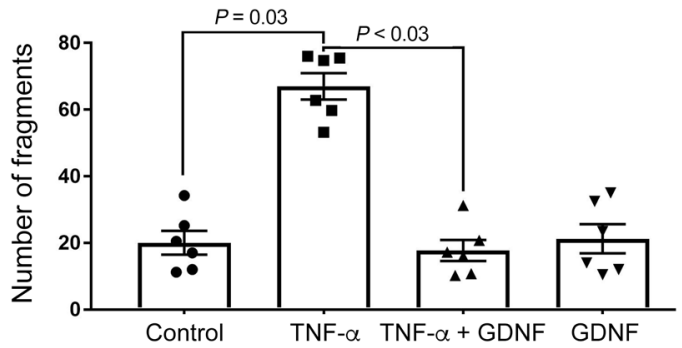

G

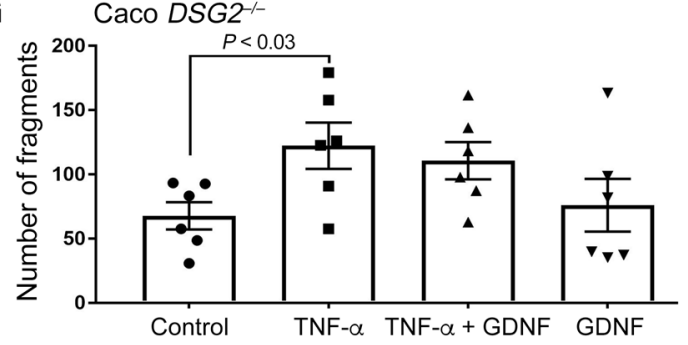

Figure 3. GDNF attenuated inflammation-induced effects in Caco2 cells. (A) In TER measurements application of TNF- $\alpha$ resulted in reduction of TER which was attenuated by simultaneous application of GDNF ( $n=6$ experiments for each condition). ${ }^{*} P<0.05$ compared with control; unpaired $t$ test for each time point. (B and C) Permeability measurements across Caco2 DSG2 ${ }^{+/+}$(B) and Caco2 DSG2 $2^{-/}$(C) were carried out under the different conditions, and permeability coefficient $\left(P_{E}\right)$ was calculated (ordinary 1-way ANOVA; $n=8-10$ for each condition). (D) Immunostaining was performed in Caco2 cells for DSC2 and for cytokeratin 18 . Images are representative of $n=6$ experiments. Scale bar is $20 \mu \mathrm{m}$ in the left (overview) and $5 \mu \mathrm{m}$ in the right panels. (E) Western blot analyses were performed in Caco2 cells for p38 MAPK, cytokeratin 18, and cytokeratin 8 and their phosphorylated forms $(n=6)$. ${ }^{*} P<0.05$ versus control; ${ }^{*} P<0.05$ versus TNF- $\alpha$ (ordinary 1 -way ANOVA). (F and G) Dispase-based enterocyte dissociation assays were performed in Caco DSG2 ${ }^{+/+}$transfected with nonsense plasmids or Caco $D S G 2^{-/-}(n=6$ for each condition; ordinary 1-way ANOVA). OD values normalized to $\beta$-actin or to total p38 MAPK, cytokeratin 18, or cytokeratin 8 are indicated below the Western blots. 
ure 4B). Simultaneous application of TNF- $\alpha$ and GDNF attenuated phosphorylation of p38 MAPK while GDNF alone reduced phosphorylation of p38 MAPK below baseline levels (Figure 3E, Figure 4B, and Supplemental Figure 5, A and B).

Because a correlation of phosphorylated cytokeratins with intestinal barrier function was suggested previously (22), phosphorylation of cytokeratins 18 and 8 was analyzed following treatment with TNF- $\alpha$ or TNF- $\alpha$ and GDNF, respectively. Application of TNF- $\alpha$ resulted in augmented phosphorylation of cytokeratin 18 at serine 52 and cytokeratin 8 at serine 74 in Caco 2 cells (Figure $3 \mathrm{E}$ and Supplemental Figure 5, C and E) and in enteroids (Figure $4 \mathrm{~B}$ and Supplemental Figure 4, B, D, and F). In contrast, no changes of phosphorylation patterns were detected for cytokeratin 18 at serine 33 (Supplemental Figure 6, A and B). Application of TNF- $\alpha$ and GDNF blocked phosphorylation of both cytokeratin 18 and cytokeratin 8 (Figure 3E, Figure 4B, and Supplemental Figure 5, $\mathrm{C}-\mathrm{F})$. Further experiments showed that increased phosphorylation of cytokeratins 18 and 8 by TNF- $\alpha$ was inhibited by simultaneous treatment of cells with p38 MAPK inhibitor SB202190. Application of SB202190 alone resulted in reduced phosphorylation of cytokeratins 18 and 8 below control levels (Figure 4C). Vice versa, activation of p38 MAPK by incubating Caco 2 cells with anisomycin (7) resulted in increased phosphorylation of cytokeratins 18 and 8 . These experiments demonstrate that cytokeratin phosphorylation in intestinal epithelial cells is mediated by activation of p38 MAPK.

In Caco 2 monolayers, application of TNF- $\alpha$ and SB202190 blocked TNF- $\alpha$-induced loss of DSG2 at the cell border (Figure $4 \mathrm{D}$ ), and cytokeratin retraction as revealed by cytokeratin 18 immunostaining was no longer detectable (Figure 4D). While treatment of cells with SB2021090 alone did not induce visible alterations of DSG2 and cytokeratin staining (Figure 4D), application of anisomycin to activate p38 MAPK showed a strong increase in phosphorylation of both cytokeratins 18 and 8, which resulted in loss of DSG2 at the cell border in immunostaining and cytokeratin retraction, comparable to the effects observed after TNF- $\alpha$ treatment (Figure 4D).

Effects of GDNF in enterocytes are mediated via the RET receptor. For neuronal cells, it is well established that GDNF mediates its effects mainly via the tyrosine kinase receptor RET (23). To assess whether this is also the case in enterocytes, we first confirmed that the RET receptor and its coreceptors GFR $\alpha$ 1-3 were present in Caco 2 cells, human and mouse tissue specimens, and enteroids (Supplemental Figure 6C). In Western blot analyses, we found that phosphorylation of RET at Tyr905, which is known to be required for activation of RET (24), was augmented in Caco2 cells following GDNF stimulation (Figure 5A). Knock down of RET receptor (Figure 5B) using a siRNA approach blunted both endogenous barrier maturation and GDNF-induced barrier maturation in measurements of TER in Caco2 monolayers (Figure 5C). Accordingly, the GDNF-induced increase and linearization of DSG2 at the cell border was not observed following knock down of the RET receptor (Figure 5D). In measurements of $4 \mathrm{kDa}$ FITC-dextran flux, increased permeability following TNF- $\alpha$ application was not blocked by GDNF after knock down of the RET receptor (Figure 5E). Similarly, under these conditions GDNF did not block augmented phosphorylation of p38 MAPK following incubation with
TNF- $\alpha$ (Figure 5F). Moreover, knock down of RET augmented basal phosphorylation of p38 MAPK (Figure 5, B and F). In summary, these experiments demonstrate that GDNF effects via p38 MAPK in enterocytes are mediated by RET-dependent signaling.

GDNF attenuates loss of IEB and inflammation in DSS-induced colitis. To determine the effects of GDNF on IEB in an in vivo model of intestinal inflammation, we induced acute colonic injury with $2.5 \%$ dextran sulfate sodium (DSS) in mice. C57Bl/6 mice (n $=18$ ) received $2.5 \%$ DSS in autoclaved drinking water ad libitum, whereas control mice received normal drinking water. One group (DSS + GDNF; $n=9$ ) was treated daily with $5 \mu \mathrm{g} / \mathrm{kg}$ bodyweight of GDNF in $100 \mu \mathrm{l} 0.9 \%$ sodium chloride intraperitoneally whereas the other group (DSS) received $100 \mu \mathrm{l} 0.9 \%$ sodium chloride. The dose of GDNF was chosen according to previous data (19) and the effective administration of GDNF was verified in ELISA-based measurements of GDNF serum concentrations and in tissue lysates of the colon after 1 hour, 8 hours, and 24 hours (Figure 6, $\mathrm{A}$ and $\mathrm{B}$ ). The baseline levels of GDNF were in the same range as reported previously for mice and shown here for humans (Figure $1 \mathrm{~B}$ and ref. 25). In this group, GDNF administration alone had no obvious side effects on body weight and stool consistency, which conforms to previous observations (26).

Following DSS application, the disease activity index (DAI) and the stool index were increased to $2.11 \pm 0.26$ and to $1.78 \pm 0.22$ after 5 days, respectively (Figure 6, C and D). Both were reduced when animals were treated with GDNF (Figure 6, B and C). In the control group, the colon length was $70.05 \pm 1.4 \mathrm{~mm}$ which was reduced to $49.11 \pm 0.84 \mathrm{~mm}$ in the DSS group. The reduction of colon length was attenuated by GDNF application $(55.1 \pm 1.7 \mathrm{~mm}$; Figure 6E). The bodyweight was reduced in the DSS group compared with controls, and it was not improved by GDNF treatment (Figure 6F). GDNF levels were not reduced in DSS-induced colitis compared with controls (Supplemental Figure 6D).

Histological analyses of $\mathrm{H} \& \mathrm{E}$-stained sections revealed an acute inflammation of the colon following DSS administration with an inflammation score of $6.7 \pm 0.8$ (Figure 7, A and B). Treatment of animals with GDNF resulted in a visible reduction of inflammation (Figure 7A), which was reflected by a significant reduction of the inflammation score (Figure 7B). Animals with DSS-induced colitis showed significant loss of DSG2 at the cell border, which was attenuated by treatment of DSS animals with GDNF (Figure 7A). Compared with controls, cytokeratin 18 staining was deranged in DSS-treated animals whereas treatment with GDNF resulted in regular staining patterns for cytokeratin 18 (Figure 7A). The tight junction protein claudin 1 was also reduced following DSS-induced colitis, which was attenuated by GDNF treatment (Figure 7), whereas E-cadherin staining was largely unaffected in acute DSS-induced colitis and by GDNF treatment (Figure 7).

Intestinal permeability as revealed by measurements of $4 \mathrm{kDa}$ FITC-dextran flux across the IEB was increased to 9.9 \pm 1.3 -fold of controls (Figure 7C). Treatment of animals with GDNF (DSS+GDNF) attenuated inflammation-induced loss of the intestinal barrier. In Western blot analyses, phosphorylation of p38 MAPK and cytokeratin 18 was increased in DSS-induced colitis, and both were attenuated by treatment with GDNF (Figure 7D and Supplemental Figure 7). 
A
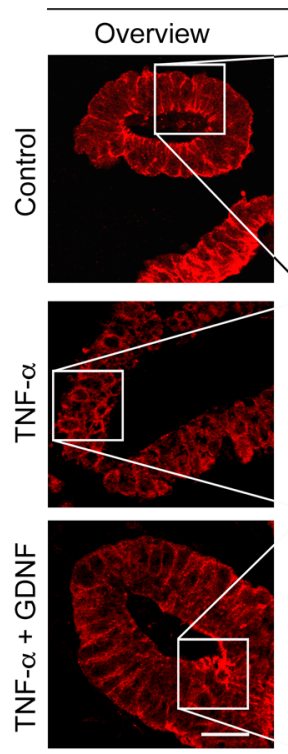

B

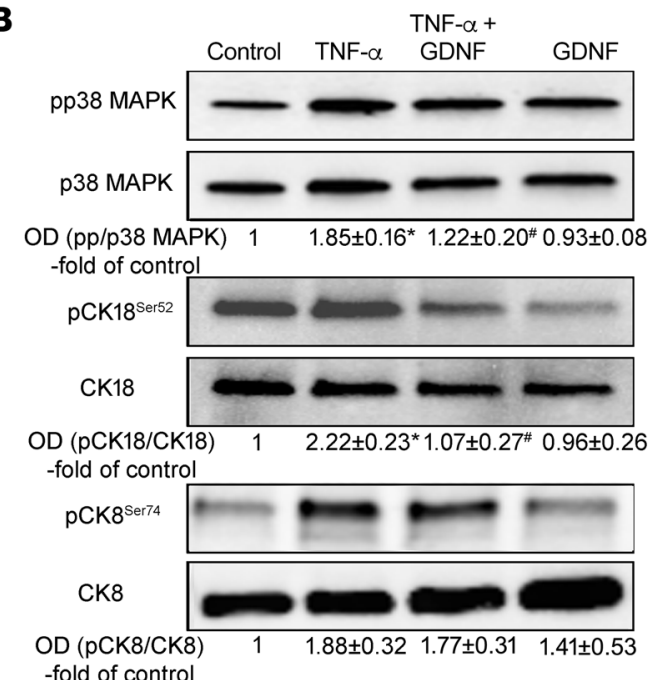

DSG2
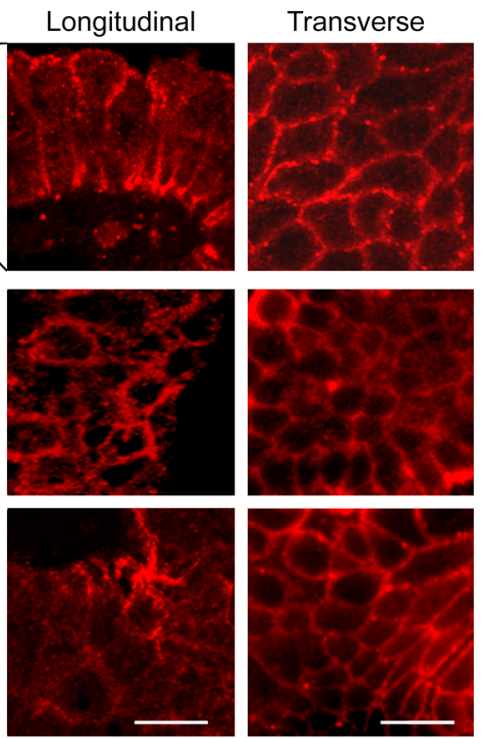

Transverse
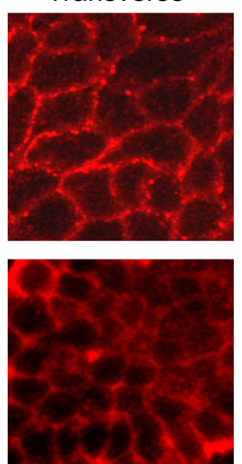

C

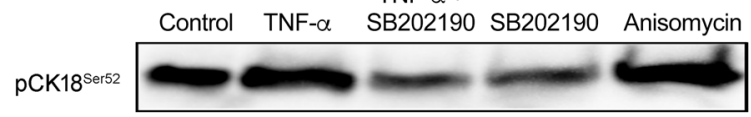

CK18

$\mathrm{OD}$ (pCK18/CK) $1 \quad 1.13 \pm 0.10 \quad 0.98 \pm 0.22 \quad 0.64 \pm 0.08^{*} \quad 8.30 \pm 1.07^{*}$ -fold of control

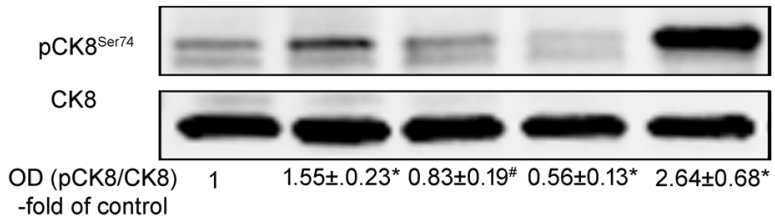

D

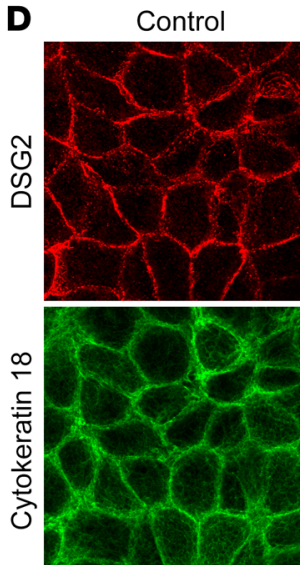

TNF- $\alpha$

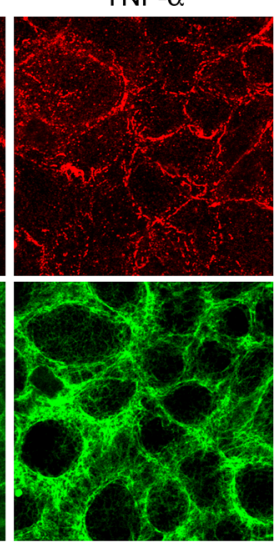

TNF- $\alpha+$ SB202190

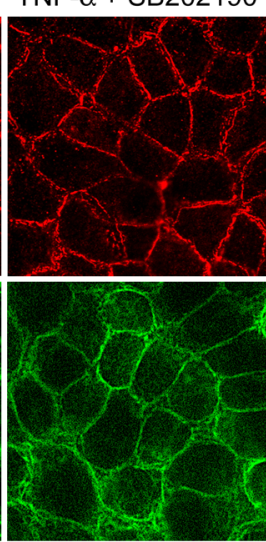

SB202190

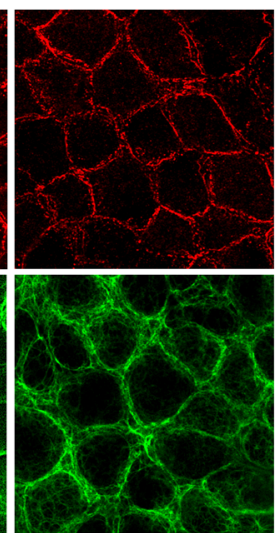

Anisomycin

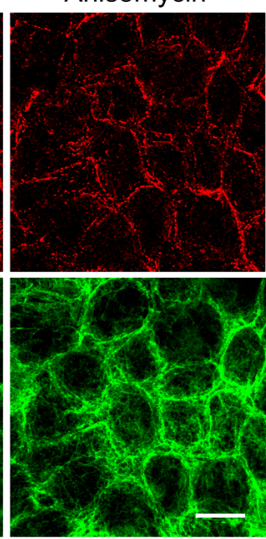

Figure 4. GDNF effects in human enteroids and TNF- $\alpha$-induced cytokeratin phosphorylation are p38 MAPK dependent. (A) Immunostaining for DSC2 and cytokeratin 18 in human enteroids. Images are representative of $n=7$ experiments. Scale bars: $20 \mu \mathrm{m}$ in the left overview panels and $7.5 \mu \mathrm{m}$ in the longitudinal/transverse panels. (B) Western blots of p38 MAPK, cytokeratin 18, and cytokeratin 8 from human enteroids are shown $(n=6) .{ }^{*} P<0.05$ compared with control; $\# P<0.05$ compared with TNF- $\alpha$ (ordinary 1-way ANOVA). (C) Western blots for cytokeratin 18 and cytokeratin 8 were performed in Caco2 cells $(n=6)$. ${ }^{*} P<0.05$ compared with control; ${ }^{*} P<0.05$ compared with TNF- $\alpha$ (ordinary 1 -way ANOVA). OD values normalized to $\beta$-actin or total p38 MAPK, cytokeratin 18, or cytokeratin 8 are indicated below the Western blots. (D) Immunostaining of DSG2 and cytokeratin 18 in Caco2 monolayers; images are representative of $n=8$ experiments. Scale bar: $20 \mu \mathrm{m}$. 


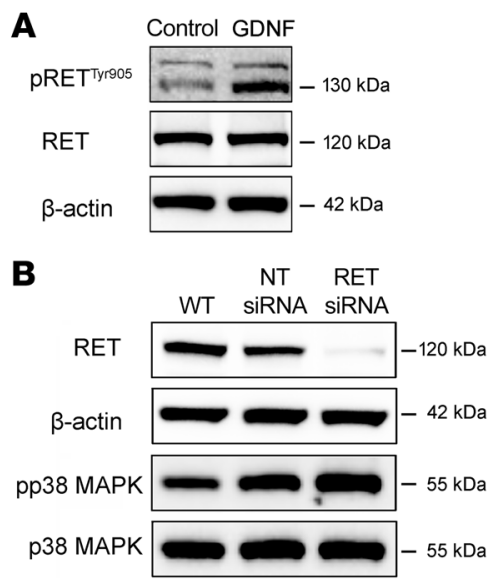

D

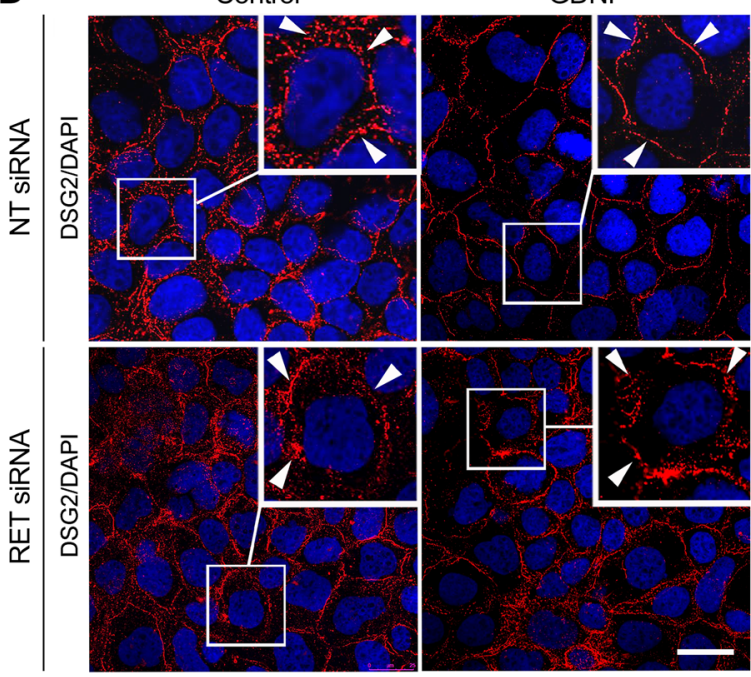

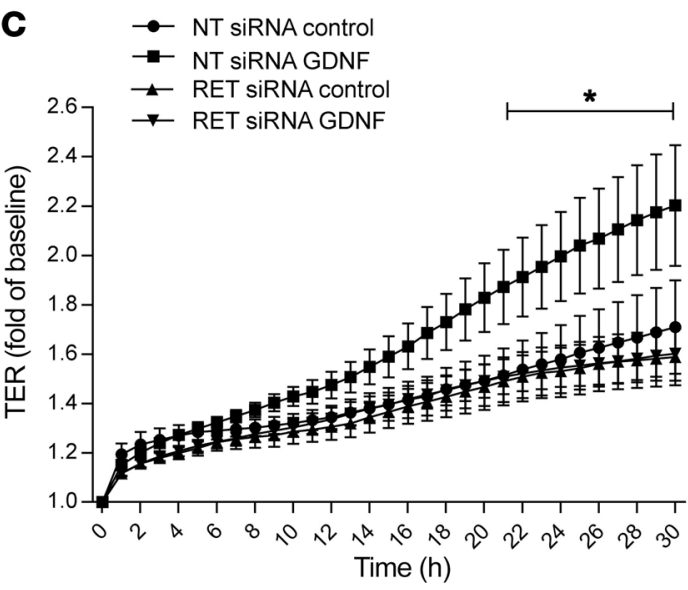

$\mathbf{E}$
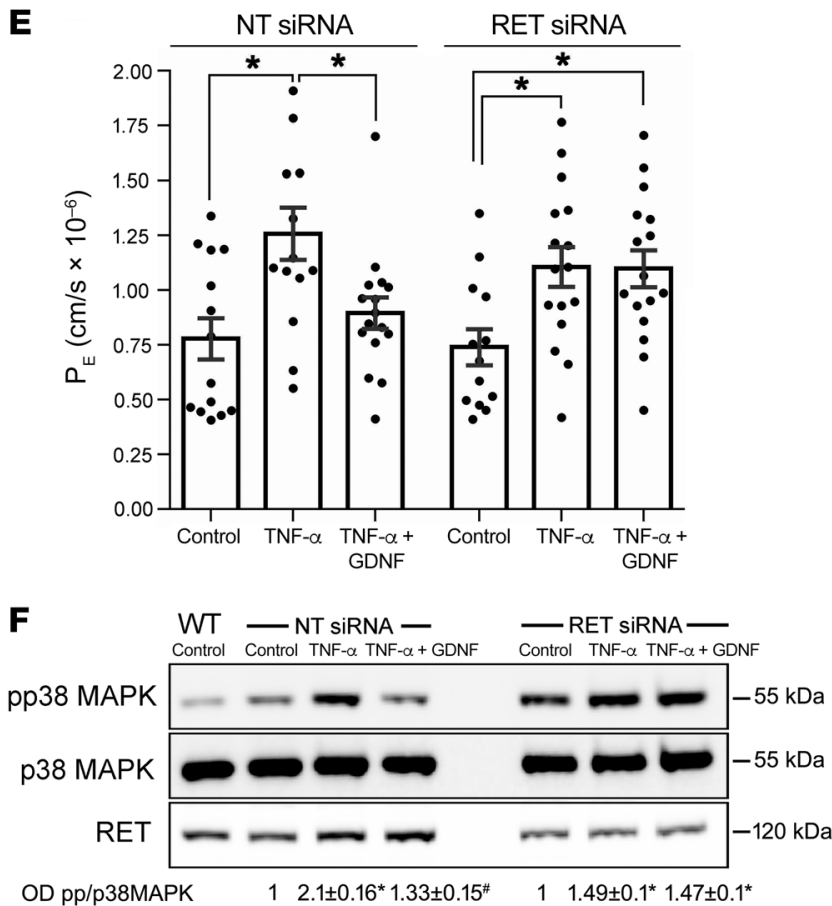

Figure 5. GDNF effects in enterocytes are RET dependent. (A and B) Western blots were performed for pRETTrr905, RET, $\beta$-actin, RET, $\beta$-actin, pp38 MAPK, or p38 MAPK under the different conditions $(n=5)$. (C) TER measurements in Caco2 cells following knock down of RET and in Caco2 transfected with nontarget siRNA (NT) are shown ( $n=8$ for each condition). ${ }^{*} P<0.05$ compared with control (unpaired $t$ test for each time point). (D) Immunostaining against DSG2 (red) and DAPI to visualize cell nuclei in Caco2 cells transfected with nontarget (NT) siRNA or RET siRNA with and without application of GDNF. Scale bar: $20 \mu \mathrm{m}$; experiments are representative of $n=8$ experiments. (E) Permeability measurements of 4-kDa FITC-dextran in Caco2 cells transfected with nontarget (NT) siRNA or RET siRNA were taken under the different conditions and permeability coefficient $\left(P_{\mathrm{E}}\right)$ was calculated (ordinary 1-way ANOVA; $n=$ 12-16 for each condition). (F) Western blots for RET, pp38 MAPK, or p38 MAPK under the different conditions are shown. OD values normalized to total p38 MAPK are indicated below the Western blots. ${ }^{*} P<0.05$ compared with control; $\# P<0.05$ compared with TNF- $\alpha$ (ordinary 1-way ANOVA; $n=8$ ).

\section{Discussion}

In the present study, we found that the neurotrophic factor GDNF was reduced in samples from patients with IBD, which correlated with the loss of desmosomal DSG2 and alterations of the intermediate filament system (Table 2). In vitro experiments in Caco2 cells revealed that GDNF acts via stabilization of DSG2 since Caco2 cells deficient for DSG2 were unresponsive to GDNF. In Caco2 cells as well as in human enteroids, GDNF protected against inflammation-induced loss of DSG2 and breakdown of the IEB. Furthermore, inflammation-induced loss of IEB by application of TNF- $\alpha$ was mediated by activation of p38 MAPK, paralleled by phosphorylation of cytokeratins 8 and 18 and a reduction of DSG2 at the cell border. This represents a new mechanism for IEB breakdown in inflammation. Evidence in support of regulation of apoptosis by GDNF as a mediator of its protective effect on the intestinal epithelium was not obtained. Rather, protective effects of GDNF on IEB were mediated by inhibiting TNF- $\alpha$-induced phosphorylation of p38 MAPK and cytokeratins via the RET receptor. The relevance of this mechanism in vivo was confirmed in a murine model of DSS-induced colitis where we observed increased phosphorylation of p38 MAPK and cytokeratins followed by loss of DSG2. Breakdown of the IEB and all other inflammation-induced 
A

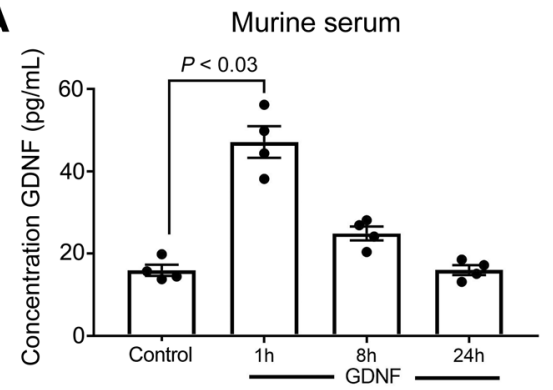

C

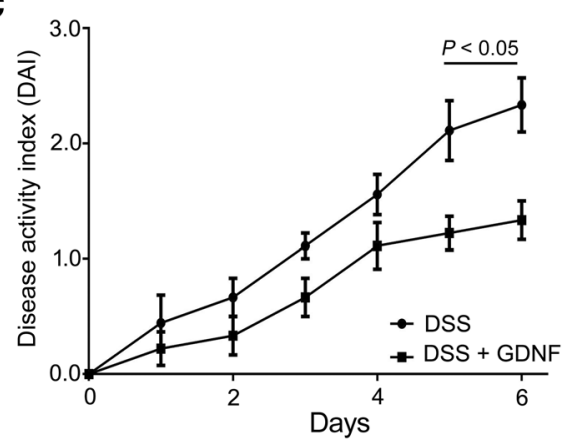

$\mathbf{E}$

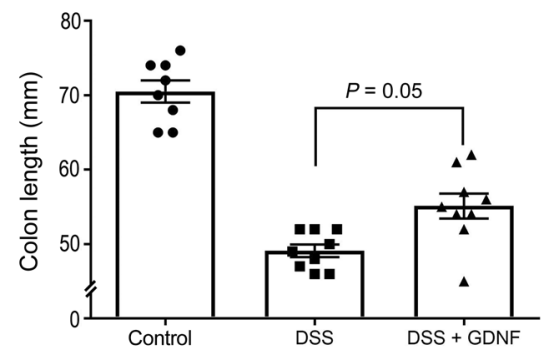

B

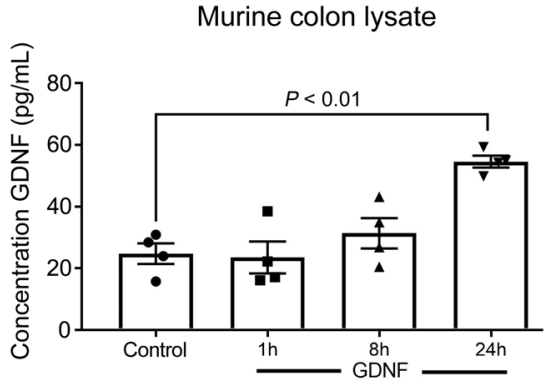

D

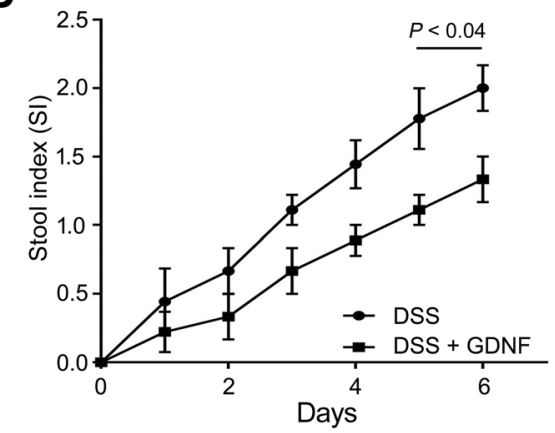

$\mathbf{F}$

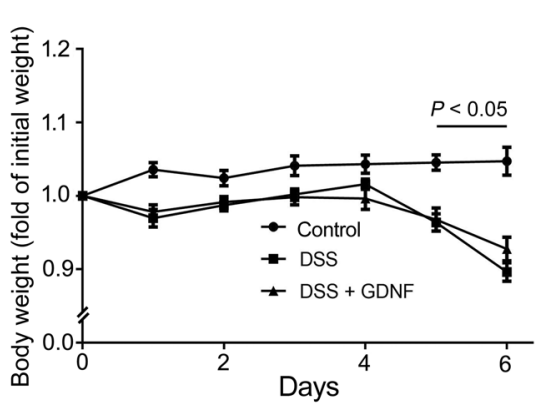

Figure 6. GDNF reduced the disease activity index in murine DSS-induced colitis. (A and B) ELISAbased measurements of GDNF levels in serum (A) and in colon lysates (B) are shown following i.p. application of GDNF in mice ( $n=4$; Kruskal-Wallis test, ANOVA). DAI (C), stool index (D), colon length $(\mathbf{E})$, and changes of body weight (F) for DSS animals are shown ( $n=9$ in each group; unpaired $t$ tests for each time point; ordinary 1-way ANOVA).

effects were attenuated by therapeutic administration of recombinant GDNF. Finally, the tight association of all these findings, i.e., the loss of GDNF and DSG2 alongside the increased phosphorylation of p38 MAPK and cytokeratins in samples from patients with $\mathrm{CD}$ and UC, point to a disease-relevant role and a mechanism of GDNF-dependent effects in IBD. The relevance of RET receptordependent signaling of GDNF in enterocytes is also shown in our present data. In summary, these findings relate to exacerbation of IBD or continued inflammatory activity. These findings do not show that GDNF signaling relates to the baseline elevated intestinal permeability in patients with $C D$ and their families.

Loss of GDNF in IBD contributes to inflammation-induced breakdown of the IEB. Our data revealed that GDNF is significantly reduced in samples from patients with CD and UC. Overall, this observation supports previous experimental models where loss of GDNF led to morphological and functional abnormalities of IEB similar to those seen in patients with IBD (17). Previously, GDNF was found to be differentially expressed throughout the intestinal tract with the highest concentrations in the colon (27). This was also in the course of IBD, and may be influenced by the state of inflammation. On the other hand, GDNF may only hold therapeutic value to promote mucosal healing at earlier stages of the disease. This hypothesis remains to be investigated in a dedicated observational clinical study.

The finding that uninflamed parts of the terminal ileum of patients with CD showed no reduction of GDNF compared with healthy controls argues against a primary loss of GDNF in the context of IBD at first glance. Therefore, it can be concluded that changes in GDNF do not relate to increased intestinal permeability under basal conditions in IBD but rather plays a role in inflammation. The finding that loss of GDNF was not observed in DSS-induced colitis in mice can be explained by the fact that the DSS model is a chemically induced model of IBD which does not reflect the pathogenesis but rather the phenotype of IBD. This strengthens our finding that, in human specimens, there is a specific role for GDNF in the pathogenesis of inflammation-induced intestinal barrier disruption in IBD. Furthermore, the fact that application of recombinant GDNF attenuated inflammation-induced loss of 
A
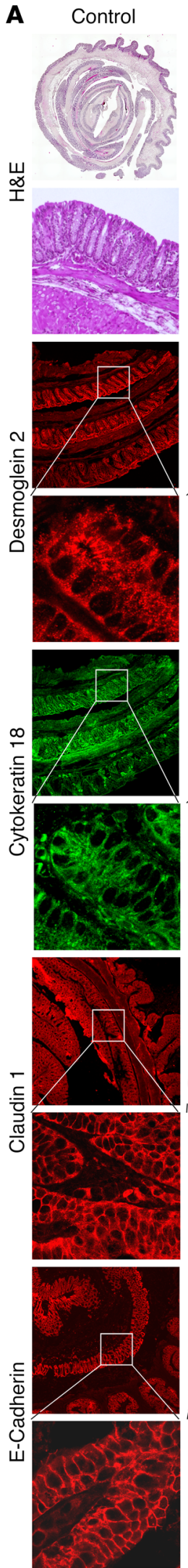

DSS
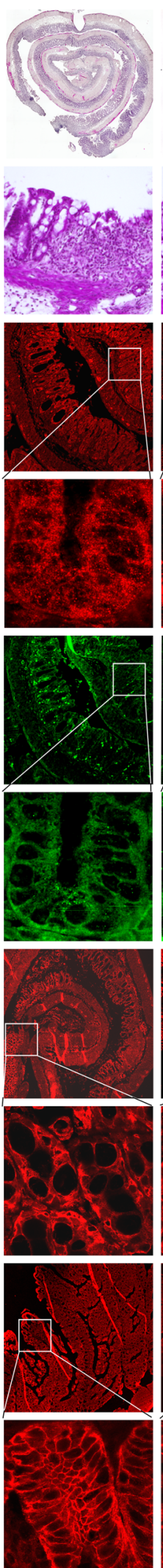

$\mathrm{DSS}+\mathrm{GDNF}$
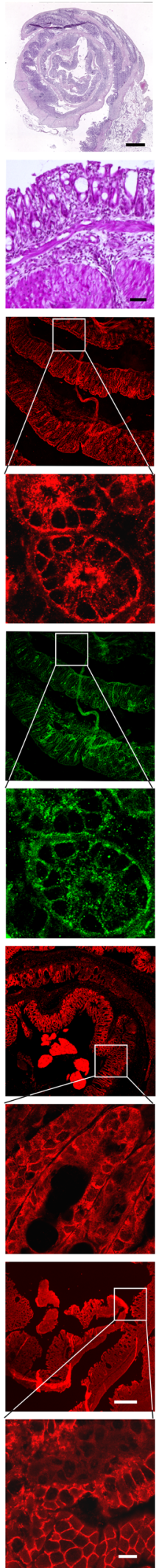

B

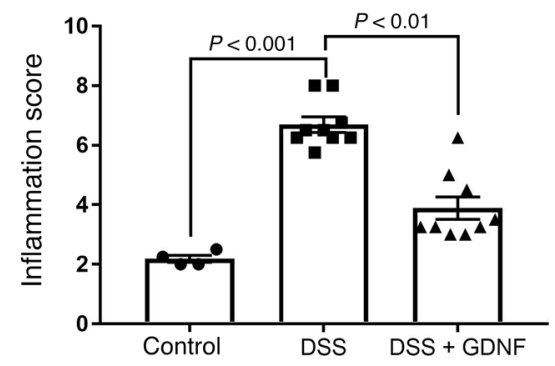

\section{C}

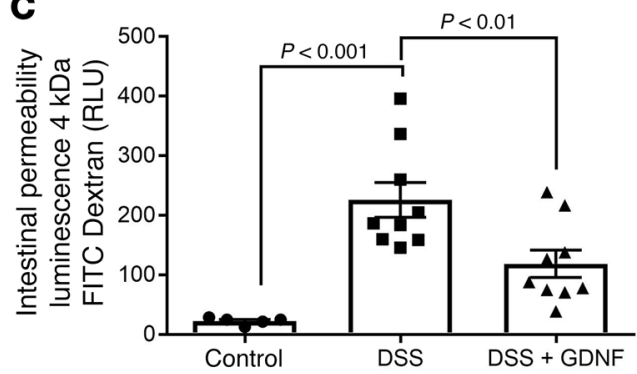

D

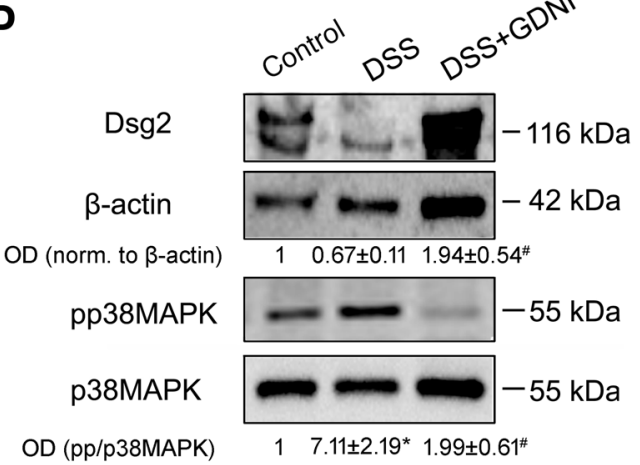

pCK18(Ser52)

CK18

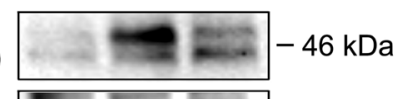

$\mathrm{OD}(\mathrm{pCK} 18 / \mathrm{CK} 18)$

pCK8(Ser74)

CK8

$\mathrm{OD}(\mathrm{pCk} 8 / \mathrm{CK} 8)$

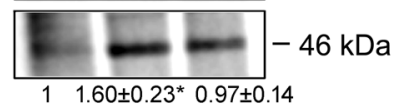

Figure 7. GDNF stabilizes intestinal barrier function in DSS-induced colitis. (A) H\&E staining of colon sections from mice are shown for controls (left), DSS-induced colitis (middle), and DSS-induced colitis with GDNF treatment (right). Immunostaining of DSC2, cytokeratin 18 , claudin 1 , and $\mathrm{E}$-cadherin are shown under the different experimental conditions. Scale bars: $100 \mu \mathrm{m}$ in H\&E overview; $50 \mu \mathrm{m}$ in the immunostaining overview panels; $20 \mu \mathrm{m}$ in all other panels. Experiments shown are representative of $n=9$ for each group. (B) Inflammation score was evaluated by H\&E staining ( $n=9$ for each group; ordinary 1-way ANOVA). (C) Intestinal permeability was measured by translocation of 4-kDa FITC-dextran from the intestinal lumen into the blood ( $n=9$ for each group; ordinary 1-way ANOVA). (D) Western blots from colon tissue lysates of animals for p38 MAPK, cytokeratin 8 , and cytokeratin 18 are shown. OD values normalized to $\beta$-actin or to total p38 MAPK, cytokeratin 18, or cytokeratin 8 are indicated below the Western blots ( $n=6$ for each group; ordinary 1-way ANOVA). ${ }^{*} P<0.05$. 
Table 2. Overview of GDNF signaling models

\begin{tabular}{|c|c|c|c|c|}
\hline \multirow[b]{2}{*}{ Aspect of GDNF signaling } & \multicolumn{4}{|c|}{ Model used } \\
\hline & $\begin{array}{l}\text { Human specimens } \\
\text { (Crohn's disease, } \\
\text { ulcerative colitis) }\end{array}$ & $\begin{array}{c}\text { Caco2 cells } \\
\text { (TNF- } \alpha \text {-induced } \\
\text { inflammation) }\end{array}$ & $\begin{array}{c}\text { Enteroids } \\
\text { (TNF-o-induced } \\
\text { inflammation) }\end{array}$ & $\begin{array}{c}\text { Mice } \\
\text { (DSS-induced colitis) }\end{array}$ \\
\hline GDNF receptors & + & + & + & + \\
\hline Reduction of GDNF concentration in inflammation & + & NA & NA & - \\
\hline RET-dependent phosphorylation of p38 MAPK & NA & + & NA & NA \\
\hline Changes of DSC2 & + & + & + & + \\
\hline Inflammation-induced phosphorylation of p38 MAPK & + & + & + & + \\
\hline Inflammation-induced phosphorylation of cytokeratin 8 and cytokeratin 18 & + & + & + & + \\
\hline Inflammation-induced keratin retraction & + & + & + & + \\
\hline Changes in permeability & NA & + & NA & + \\
\hline Mechanical stability & NA & + & NA & NA \\
\hline Antiapoptotic effects of GDNF & NA & + & NA & + \\
\hline
\end{tabular}

Overview demonstrating which aspects of GDNF signaling were studied in the different models. +, result as indicated; -, negative result; NA, experiment not performed.

barrier function underlines the importance of GDNF in this context. It can be speculated that there may be increased susceptibility of enteric glial cells leading to alterations of GDNF secretion. This is supported by previous data from biopsies of patients with IBD and animal models of IBD which consistently suggested a role of inflammatory effects on the ENS in the generation of symptoms associated with IBD (29). The substantial role for enteric glial cells and especially GDNF in the context of inflammatory response was pointed out by a study in which TLR2-deficient mice displayed disturbed architecture of the ENS, which resulted in reduced GDNF expression and breakdown of the IEB (17). In the latter study, application of GDNF led to reconstitution of the ENS but potentially direct effects of GDNF on enterocytes remained unexplored (17). In contrast to these observations, recent studies demonstrated that enteric glial cells are not required for maintenance of the epithelium in mice (14) and do not acutely effect gut permeability (30). Therefore, it must be considered that in addition to alterations of enteric glial cells in IBD, smooth muscle cells in the gut wall and even enterocytes represent an additional source of GDNF, and both may be critically involved in the regulation of intestinal GDNF levels in health and disease $(15,16)$. This idea conforms to the observation that the sole increase of epithelial GDNF in specimens of patients with IBD may be part of a rescue mechanism following inflammation (18). The most important source of GDNF is thought to be the ENS, although GDNF may also derive from smooth muscle cells or enterocytes. This remains to be determined in future studies as it was not in the scope of the present study.

GDNF attenuated all inflammation-induced effects in intestinal epithelial cells via the RET receptor. In previous studies and here, we and other groups confirmed the presence of GDNF receptors and responsiveness to GDNF in enterocytes $(16,18)$. Furthermore, we demonstrate here for what we believe is the first time that GDNF effects in enterocytes require RET receptor-dependent signaling. On the one hand, GDNF augmented phosphorylation of the RET receptor. On the other hand, loss of the RET receptor by siRNA blunted all GDNF-induced effects in enterocytes. Interestingly, in a nationwide population-based cohort study from Sweden, individuals with Hirschsprung's disease (HD) showed an increased risk to develop IBD with an odds ratio of 4.99 (31). However, a metaanalysis that was published around the same time showed that only a subset of male patients with HD after the surgical Duhamel procedure with ongoing enterocolitis have an association with IBD, especially CD (32). Based on these observations, a clinical relevance for our observations can be assumed.

Previous studies suggested that GDNF indirectly stabilizes the IEB by inducing antiapoptotic effects on colonic enterocytes and immunomodulation $(18,19,33,34)$. According to our in vitro data in Caco 2 cells and in human enteroids, application of GDNF resulted in direct effects on enterocytes, which prevented inflammation-induced loss of IEB. A potential role of apoptosis induced by TNF- $\alpha$ under the experimental conditions used here in vitro was excluded and therefore GDNF apparently did not exert its protective influence via antiapoptotic effects. In vivo, we clearly detected apoptosis as revealed by activated caspase 3 in colon lysates of animals with DSS-induced colitis. This, however, was not blocked markedly by GDNF treatment, showing that protective effects of GDNF are not mediated by blocking apoptosis in vivo.

For what we believe is the first time, we provide evidence that GDNF effects are mediated by strengthening DSG2 function, which was proven by the fact that DSG2-deficient cells showed no response to GDNF. Based on our in vitro data where no immune cells were present, we assume that DSG2 has no immunomodulatory role itself but exerts its effects by stabilization of intercellular junctions in enterocytes. The effects on DSG2 in enterocytes by GDNF were substantiated by immunostaining, triton-extraction, and AFM, in which application of GDNF resulted in increased DSG2 at the cell border and augmented DSG2-mediated adhesion. Furthermore, inflammation-induced loss of DSG2 at the cell border was restored by GDNF in vivo and in vitro which resulted in increased cell-cell adhesion in dispase-based enterocyte dissociation assays and augmented binding activities in AFM measurements. In DSG2-deficient Caco2 cells, GDNF failed to restore TNF- $\alpha$-induced loss of cell-cell adhesion underlining 
the importance of DSG2 in this context. In summary, these data not only show a novel target of GDNF-induced effects in enterocytes, but also support the growing evidence of the importance of desmosomal integrity in maintaining the IEB in health and disease $(7,35-38)$. The clinical evidence about the role of DSG2 demonstrates that patients with DSG2 mutations have a cardiac phenotype known as arrhythmogenic right ventricular dysplasia/ cardiomyopathy (39). However, an intestinal phenotype in these patients has not been described. An explanation for this may be that DSG2 mediates tissue-specific signaling events in addition to strengthening of intercellular adhesion. In cardiomyocytes, DSG2 and the plaque protein Desmoplakin were shown to directly regulate gap junctions so that mutations of both contribute to the severe cardiac phenotype $(40,41)$. In enterocytes, DSG2 regulates other signaling pathways including cell proliferation, cell death, and tight junction integrity $(7,38,42)$. These may be compensated under basal conditions when DSG2 mutations are present, which may explain the missing intestinal phenotype. This is supported by previous data in mice that showed total loss of intestinal DSG2 led to increased susceptibility in inflammation but was largely compensated under basal conditions (8). This observation and the loss of DSG2 in patients with IBD underline the relevance for DSG2 to maintain intestinal barrier integrity in inflammation $(7,8)$. It has to be pointed out that tight junction proteins are also greatly affected by inflammation as was also observed here in mice with DSS-induced colitis, whereas E-cadherin was largely unaffected. This demonstrates that enterocytes were present despite the presence of severe inflammation. In addition, this supports the view that mechanical stabilization of intercellular adhesion by DSG2 is a necessary prerequisite for stabilization of tight junctions.

p38 MAPK-dependent phosphorylation of intermediate filaments induces loss of IEB in inflammation. Regulation of desmosomal adhesion has been extensively investigated in the context of the skin-blistering disease pemphigus in keratinocytes where autoantibody-induced loss of desmoglein3-mediated (Dsg3-mediated) adhesion results in activation of p38 MAPK and keratin filament reorganization (9). Additionally, it was recently shown that adhesive forces of Dsg3 in keratinocytes are mediated by keratin-dependent regulation of $\mathrm{p} 38 \mathrm{MAPK}$ (43). Our present data demonstrate that p38 MAPK was activated in both UC and CD, which correlated with loss of DSG2. We detected loss of DSG2 in CD in our previous study (7), but it was not previously described for patients with UC. The barrier destabilizing effect of p38 MAPK activated in response to TNF- $\alpha$ in intestinal epithelium is also supported by our previous study in which inflammatory stimuli induced strong activation of $\mathrm{p} 38$ MAPK followed by loss of DSG2-mediated adhesion (7), so that p38 MAPK activation appears to be detrimental for intestinal barrier integrity. On the other hand, it was reported that a proper balance of $\mathrm{p} 38$ MAPK activation is important since activation of p38 MAPK was also required for barrier recovery following $\mathrm{Ca}^{2+}$-depletion in a cell culture model of enterocytes (20). This emphasizes that the role of p38 MAPK in intestinal barrier regulation is not completely understood.

Following p38 MAPK activation, we observed the phenomenon of keratin retraction as has amply been described in keratinocytes after stimulation with pemphigus autoantibodies directed against Dsg3 which then led to internalization of Dsg3 (44). In the context of IBD, it was recognized earlier that patients with CD or UC have missense mutations in keratin 8, which led to the assumption that this may lead to augmented susceptibility in intestinal epithelium (45). In support of this, it was shown that interleukin 6 -dependent expression of keratin 8 protected the intestinal barrier from inflammatory stimuli (46). Cytokeratin 8 phosphorylation at ser74 has previously been identified as a substrate for phosphorylation by p38 MAPK and it was observed that under certain conditions of inflammation, increased keratin 8 phosphorylation occurs, which was linked to increased susceptibility of the IEB by unknown mechanisms (22). This hypothesis is now substantiated by our present data where cytokeratin 8 and 18 phosphorylation is indeed critically involved in the process of IEB regulation most likely by affecting desmosomal integrity. In general, it is known that phosphorylation of keratins changes the distribution of keratins in the cell, alters its polymerization behavior, and is associated with keratin granule formation $(47,48)$. Moreover, it was shown in keratinocytes that keratins control intercellular desmosomal adhesion, since desmosomes lacking anchorage to intermediate filaments by adapter proteins undergo a more rapid endocytosis with consecutive loss of intercellular adhesion (49). A comparable mechanism can be assumed based on our present data in enterocytes since phosphorylation of keratins led to retraction of the peripheral keratin ring. This may reduce tethering of DSG2 to the intermediate filaments with consecutive loss of desmosomal adhesion and intestinal barrier function.

In summary, inflammation-induced cytokeratin phosphorylation by activation of p38 MAPK, which led to loss of DSG2 as shown here in vitro, in vivo, and in the samples of patients suffering from IBD, points to a novel and important pathomechanism for barrier dysregulation in CD and UC. As outlined above, this observation relates to exacerbation of IBD or continued inflammatory activity though it does not explain elevated intestinal permeability in patients with IBD. Nonetheless, GDNF-induced inhibition of this pathway as outlined above not only provides what we believe are novel insights into the role of GDNF in the pathogenesis of barrier dysregulation in IBD, but also implies an interesting potential for novel therapeutic options.

\section{Methods}

Test reagents. Recombinant human GDNF (PeproTech) and TNF- $\alpha$ (Biomol) were used at $100 \mathrm{ng} / \mathrm{ml}(7,16)$. The p38 MAPK inhibitor SB202190 was used at $30 \mu \mathrm{M}$ (Calbiochem) and anisomycin (Sigma-Aldrich) was used at $60 \mu \mathrm{M}$ to activate p38 MAPK (16).

Human tissue samples. Human tissue samples were obtained from patients suffering from IBD with an indication for surgical resection. Tissue samples from patients with $\mathrm{CD}(n=9)$ derived from the terminal ileum. They were taken from the center of the inflamed parts of the resection specimens and from the periphery where no inflammation was seen. In patients with ulcerative pancolitis $(n=9)$ a sample of the affected colon was taken. Since the whole colon was inflamed in these patients, no uninflamed tissue was collected. Control tissue samples (colon or terminal ileum) from patients not suffering from IBD derived from patients that required right or left hemi-colectomy due to colon carcinoma in which the surgical resection routinely involved a part of the healthy small intestine or colon, respectively. Detailed patient characteristics are presented in Table 1. 
For Western blot and ELISA analyses, samples of the mucosa and whole gut wall were taken immediately after resection from each specimen and then snap frozen in liquid nitrogen and processed. A second part of the tissue samples was fixed in $4 \%$ paraformaldehyde, embedded in paraffin, sectioned $(1 \mu \mathrm{m})$, and immunostained as previously described (7).

Cell culture. Caco2 cells (ATCC) were cultured in Eagle's minimum essential medium (EMEM; ATCC) supplemented with $50 \mathrm{U} / \mathrm{ml}$ penicillin-G, $50 \mu \mathrm{g}$ streptomycin, and 10\% FCS (Biochrom). Cultures were used for experiments when grown to confluent monolayers. For experiments, cells were serum-starved for 24 hours. Mycoplasma contamination was excluded prior the experiments.

Generation of CRISPR/Cas9-mediated gene knock out for DSG2 in Caco 2 cells. sgRNAs for SpCas9-mediated genome editing were designed using the Chopchop web-based sgRNA design tool (50). Two oligos targeting exon 3 and exon 13 of human DSG2 were designed and subcloned into 2 lentiviral vectors; sgRNA targeting exon 3 into pLentiCRISPR v2 (gift from Feng Zhang, Addgene plasmid 52961; Broad Institute of MIT and Harvard, Cambridge Center) and sgRNA targeting exon 13 in pLKO5.sgRNA.EFS.GFP (gift from Benjamin Ebert, Addgene plasmid 57822; Division of Hematology, Department of Medicine, Brigham and Women's Hospital, Harvard Medical School). Lentiviral particles were produced using HEK293T cells utilizing a second-generation lentiviral packaging system comprising pPAX and pMD2 packaging plasmids. Caco2 cells were seeded in 6-well plates and 24 hours later infected in the presence of polybrene, with viral supernatant comprising both viral particles LKO-sgRNA-EFS GFP and CrisprV2. Three days after infection, cells were selected with puromy$\operatorname{cin}(15 \mu \mathrm{g} / \mathrm{ml})$ for 1 week with medium changes every 2 days. Puromycin-positive clones were FACS sorted for GFP expression and reseeded as single cells in a 24-well plate. Single clones were propagated and loss of DSG2 was assessed in single clones by Western blot and qPCR (Supplemental Table 1).

Transfection of Caco2 cells with RET siRNA. Human RET-specific siRNA oligo pools and nontarget controls were purchased from Thermo Fisher Scientific (s11936) and Santa Cruz Biotechnology (sc-37007). Lipofectamine (Invitrogen) was used as a transfection reagent according to the manufacturer's protocols. Briefly, cells were transfected at $70 \%$ confluency and medium was exchanged after 24 hours. Experiments were conducted after 48 hours when a sufficient knockdown was established (7).

Enteroids. IECs were isolated from human full-wall gut resections $(1 \mathrm{~cm})$ from the terminal ileum of healthy control patients not suffering from IBD, as previously described (51). Villi were scraped off the mucosa using a sterile glass slide. The remaining tissue was transferred into a $50 \mathrm{ml}$ falcon tube with $20 \mathrm{ml} 4^{\circ} \mathrm{C}$ cold HBSS (Sigma-Aldrich), vortexed for 5 seconds, and the supernatant discarded. After several washing steps to clear the supernatant of cell debris, the tissue was incubated in $4^{\circ} \mathrm{C}$ cold $2 \mathrm{mM}$ EDTA/HBSS solution (Sigma-Aldrich) for 30 minutes at $4^{\circ} \mathrm{C}$ under gentle rotation on a shaker. Subsequently, the tissue was washed once in $20 \mathrm{ml}$ HBSS by manually inverting the tube 5 times. The mucosa was transferred to a new tube with $10 \mathrm{ml}$ HBSS and manually shaken 5 times. This shaking procedure was repeated 4 times always using a new tube. Each cell fraction was checked for the amount and size of crypts within small drops under the microscope. The supernatants containing the most vital-appearing crypts were pooled and centrifuged at $350 \mathrm{~g}$ for 3 minutes at room temperature.
Pellet was resuspended in $10 \mathrm{ml}$ basal medium, DMEM-F12 Advanced (Invitrogen) supplemented with N2, B27, antibiotic-antimycotic, 10 mM HEPES, 2 mM GlutaMAX-I (all from Invitrogen), 1 mM N-acetylcysteine (Sigma-Aldrich), and the crypt number was estimated in a 10 $\mu \mathrm{l}$ drop by microscopy. Crypts were centrifuged in a nonstick $1.5-\mathrm{ml}$ tube at $350 \mathrm{~g}$ for 3 minutes at room temperature and the supernatant was removed. The tube with the cell pellet was placed on ice until further use. The pellet was resuspended in 5000 crypts $/ \mathrm{ml}$ cold Matrigel (Corning). Drops of $50 \mu \mathrm{l}$ per well were seeded in a 24 -well plate and incubated for 10-20 minutes until the Matrigel was well solidified. The culture medium contained a mixture of $50 \%$ fresh basal medium and $50 \%$ Wnt3A-conditioned medium.

The following growth factors and inhibitors were added to the culture medium: $500 \mathrm{ng} / \mathrm{ml} \mathrm{hR-Spondin} \mathrm{1,} 100 \mathrm{ng} / \mathrm{ml} \mathrm{mNoggin,} 50$ $\mathrm{ng} / \mathrm{ml}$ mEGF (all from PeproTech), $10 \mu \mathrm{M}$ Y-27632 (ROCK inhibitor; Tocris Bioscience, R\&D Systems), $10 \mathrm{nM}$ Gastrin ([Leu15]-Gastrin I; Sigma-Aldrich), $10 \mathrm{mM}$ Nicotinamide (Sigma-Aldrich), $500 \mathrm{nM}$ A83-01 (Tocris Bioscience, R\&D Systems), $10 \mu \mathrm{M}$ SB202190 (SigmaAldrich), and 500 nM LY2157299 (Axon MedChem). Intestinal epithelial cells were expanded as enteroids for 3-4 weeks by adding $500 \mu \mathrm{l}$ of this medium per well. The medium was changed every second day. Enteroids for this study were chosen from an existing cryo cell bank, defrosted, cultured, and thereafter used for this study up to passage 30 .

Animal experiments. After approval by the animal care committee (Laboratory Animal Care and Use Committee of the District of Unterfranken; AZ 2-272), experiments were performed on male C57BL/6J mice (Janvier Labs). Animals were kept under conditions that complied with the NIH Guide for the Care and Use of Laboratory Animals, and studies were approved by the governments of Unterfranken and Germany. Animals were kept on a standard diet and 12-hour day and night cycles.

Experimental setup. We used DSS as a murine model for induction of colitis. Eight-week-old male mice received 2.5\% DSS in autoclaved drinking water ad libitum. Mice were monitored daily to evaluate the DAI, which included changes of body weight, stool consistency, and presence of blood in the stool using hemoccult (Care Diagnostica). The following scoring system was used to describe the stool quality (52): normal stool consistency with negative hemoccult (0), soft stools with positive hemoccult (1), very soft stools with traces of blood (2), watery stools with visible rectal bleeding (3). Animals were randomized in 2 groups. GDNF-treated animals $(n=9)$ received a solution of $100 \mu \mathrm{l} 0.9 \% \mathrm{NaCl}$ containing $5 \mu \mathrm{g} / \mathrm{kg}$ bodyweight of GDNF, whereas DSS-alone animals $(n=9)$ received only $100 \mu \mathrm{l} 0.9 \% \mathrm{NaCl}$. Injections were administered intraperitoneally and repeated every 24 hours.

Measurement of intestinal permeability and tissue harvesting. After 6 days, laparotomy was performed in anesthetized mice using isoflurane (Forene; Abbott). The colon was mobilized and opened at the ileocecal valve and at the upper rectum.

After flushing the colon with PBS at room temperature to remove blood and stool, the colon was ligated at the cut ends without compromising the blood supply. To determine intestinal permeability, $200 \mu \mathrm{l}$ of $4 \mathrm{kDa}$ FITC dextran diluted in PBS $(1 \mathrm{mg} / \mathrm{ml})$ was injected into the ligated colon. After 1 hour, blood from the inferior vena cava was taken to measure the concentration of $4 \mathrm{kDa}$ FITC dextran translocated from the colonic lumen into the blood. The blood samples were centrifuged at $17,094 \mathrm{~g}$ for 10 minutes at $4^{\circ} \mathrm{C}$, and the luminescence of the serum was quantified by using Genios Pro Reader (Tecan). After blood collec- 
tion mice were euthanized by exsanguination, the colon was harvested, and its length was measured.

The ligations of the colon were removed and the colon was cut longitudinally into 2 pieces. One part was fixed in $4 \%$ paraformaldehyde embedded in paraffin and sectioned. Two blinded investigators quantified the tissue inflammation in H\&E-stained sections of the colon using inflammation scoring (53) for extent of inflammatory cell infiltration (none $=1$, mucosal infiltration $=2$, submucosal infiltration $=3$, transmural infiltration $=4$ ) and severity of epithelial damage (no epithelial damage $=1$, focal lesions $=2$, multiple lesions $=3$, extended ulcerations $=4$ ), resulting in a total scoring range of 2 to 8 per mouse. The other half of the colon was lysed and homogenized with a Tissue Lyzer (Qiagen) in a SDS lysis buffer and used for Western blot analysis and quantification of GDNF concentrations using GDNF ELISA (Promega).

GDNF ELISA. GDNF ELISA was performed according to the manufacturer's protocol (Abnova; murine KA3041 and human KA0984). The assay is an antibody sandwich ELISA in which 96-well plates are coated with anti-GDNF monoclonal antibody, which binds soluble GDNF.

Immunostaining. Cultured cell monolayers were prepared for immunostaining as previously described (16). Epithelial cells were grown to confluence on coverslips. Human and animal tissue samples and enteroids embedded in paraffin were sectioned in $1-\mu \mathrm{m}$ slices. Immunostaining was performed after removal of paraffin as described for epithelial monolayers (7). Then monolayers and tissue slides were incubated at $4^{\circ} \mathrm{C}$ overnight using antibodies, as outlined in Supplemental Table 2. Representative experiments were photographed with a fluorescence microscope BZ-9000 (BioRevo; Keyence) and a confocal microscope (TCS SP2; Leica).

Western blot. For Western blot analyses, cells were grown on 6-well plates and homogenized in SDS lysis buffer containing $25 \mathrm{mM}$ HEPES, 2 mM EDTA, $25 \mathrm{mM} \mathrm{NaF}$, and 1\% SDS. To analyze human enterocytes, the mucosa was mechanically dissected from the underlying tissue immediately after the resection. Full-wall specimens were used for analyses of the animal experiments. Human specimens and distal murine colon were examined in SDS lysis buffer using TissueLyzer (Qiagen).

SDS gel electrophoresis and blotting were carried out after normalization of the protein amount using BCA assay (Thermo Fisher Scientific), as previously described (16). Primary and secondary antibodies were used as outlined in Supplemental Table 2 in 5\% BSA and 0.1\% Tween. Bound immunoglobulins were visualized by the enhanced chemiluminescence technique (Amersham).

Chemiluminescence signal detection and quantification were performed by densitometry (ChemicDoc Touch Bio-Rad Laboratories). Optical densities were quantified in each Western blot using Image Lab (ChemicDoc Touch Bio-Rad Laboratories) for statistical evaluation.

Cell viability/apoptosis assays. CellTiter-Glo 2.0 assay (Promega; catalog G9241), a luminescence-based cell viability assay, was used as previously described for measuring cell viability (54). In this assay, the number of viable cells in culture is determined by quantification of the amount of ATP present. In another assay, cell viability was determined by crystal violet (CV) staining (0.5\% CV in $25 \%$ methanol) as previously described (55). To detect the amount of apoptosis, propidium iodide staining was carried out and the number of propidium iodide-positive cells/high power filed was quantified as previously described in detail (56).
Membrane protein extraction assay. Protein fractionation was carried out using Mem-Per Plus Kit (Thermo Fisher Scientific). Cells were harvested in growth media by scraping them from the bottom with a cell scraper. After centrifugation at $8.05 \mathrm{~g}$ for 5 minutes and washing 3 times, cells were permeabilized with a permeabilization buffer to release the cytosolic fraction. The cytosolic fraction was separated by centrifugation at $24,371 \mathrm{~g}$ for 15 minutes. The pellet containing the membrane-associated proteins was then resuspended in a solubilization buffer. The suspension was centrifuged another time at $24,371 \mathrm{~g}$ for 15 minutes to remove particulate material. Then the cytosolic and membrane-associated supernatants were used for Western blot analysis.

Atomic force microscopy. The approach of AFM force spectroscopy on living cells was previously described in detail (21). Cells were grown on glass coverslips and treated with GDNF $(100 \mathrm{ng} / \mathrm{ml}) 1$ day before confluence or with $4 \mathrm{mM}$ EGTA for 30 minutes after finished control measurements. For analysis of DSG2 interactions on the surface of living cells, a Nanowizard III AFM (JPK Instruments) mounted on an optical microscopy (Carl Zeiss) was used. The approach of AFM force spectroscopy on living cells was previously described in detail (21). Imaging and force measurements were performed in cell culture medium using flexible $\mathrm{Si}_{3} \mathrm{~N}_{4}$ AFM cantilevers (MLCT Probes, Bruker) coated with a flexible bifunctional polyethylene glycol linker (Gruber Lab, Institute of Biophysics) and recombinant DSG2-Fc containing the complete extracellular domain (ED) of DSG2, as outlined elsewhere (57). At first, AFM topography images of $50 \times 50 \mu \mathrm{m}$ and $128 \times 128$ pixels were created using a force curve-based imaging mode (QI-mode) with a setpoint adjusted to $0.5 \mathrm{nN}$, a z-length of $1.5 \mu \mathrm{m}$, and a pulling speed of $50 \mu \mathrm{m} / \mathrm{s}$. For adhesion measurements, a small area of $2 \times 5$ $\mu \mathrm{m}$ was selected and 1000 force distance curves were recorded for each area using the force mapping mode with a relative setpoint of 0.5 $\mathrm{nN}$, a z-length of $3 \mu \mathrm{m}$, and a pulling speed $5 \mu \mathrm{m} / \mathrm{s}$. JPK data processing software (JPK Instruments) was applied for processing AFM images and analysis of force distance curves and Origin 9.1 (Originlab) was used for peak fit analysis of measured unbinding force curves.

Dispase-based enterocyte dissociation assays. As previously described (7), confluent cells in 24-well plates were exposed to the test reagents as indicated below, washed with HBSS (Sigma-Aldrich) and incubated with Dispase-II (Sigma-Aldrich) for 30 minutes to release the monolayer from the well bottom. Afterwards, the cell sheet was exposed to shear stress by pipetting 5 times. Four fields of view were photographed with BZ-9000 (BioRevo, Keyence) and numbers were quantified.

Measurements of transepithelial electrical resistance. To measure transepithelial electrical resistance (TER) we used the ECIS TransFilter Adapter for ECIS 1600R across cell monolayers (Applied Biophysics, Ibidi). Cells were seeded on 24-well transwell chambers and measurement was started immediately. At confluency of monolayers, cells were treated with or without mediators as indicated.

Measurement of FITC-dextran flux across monolayers of cultured epithelial cells. As previously described (15), epithelial cells were seeded on top of transwell chambers on 6-well plates $(0.4 \mu \mathrm{m}$ pore size; Falcon) and grown to confluence. After rinsing with PBS, cells were incubated with fresh DMEM without phenol red (MilliporeSigma) containing $10 \mathrm{mg} / \mathrm{ml}$ FITC-dextran $(4 \mathrm{kDa}$ or $70 \mathrm{kDa})$ in the presence or absence of different mediators. Paracellular flux was assessed by taking 100- $\mu$ l aliquots from the outer chamber over 2 hours of incubation. Fluorescence was measured using a Wallac Victor 2 fluorescence 
spectrophotometer (Tecan GENios Microplate Reader, MTX Lab Systems) with excitation and emission at $485 \mathrm{~nm}$ and $535 \mathrm{~nm}$, respectively. For all experimental conditions, permeability coefficients $\left(\mathrm{P}_{\mathrm{E}}\right)$ were calculated as previously described (15).

Statistics. Statistical analysis was performed using Prism (GraphPad Software). Data are mean \pm SE. Statistical significance was assumed for $P$ less than 0.05. To check for normal distribution we used either D'Agostino and Pearson normality test or Shapiro-Wilk test, depending on the sample size. Parametric data were analyzed using unpaired $t$ test or ordinary 1-way ANOVA followed by Bonferroni's correction. For nonparametric data we used the Mann Whitney $U$ test for paired experiments or the Kruskal-Wallis test followed by Dunn's test (ANOVA) for multiple experiments. The tests used for the individual experiments are indicated in the figure legends.

Human and animal study approval. Prior to surgery, all patients gave written informed consent for inclusion in the study. Ethical approval was given by the ethical board of the University of Wuerzburg (proposal numbers 113/13, 46/11, 42/16). Animal experiments were carried out by the Animal Research: Reporting of In Vivo Experiments (ARRIVE) guidelines. Animal care committee approval (Laboratory Animal Care and Use Committee of the district of Unterfranken; AZ 2-272) was obtained before experiments started.

\section{Author contributions}

M Meir, NB, and NS contributed to the study concept and experiments, drafted figures, and wrote the manuscript. HU contributed to experiments, drafted figures, and wrote the manuscript. MD contributed to experiments and drafted the manuscript. SF contributed to the study concept and experiments, and drafted the manuscript. FK contributed to the experiments. CTG contributed to the study concept and drafted the manuscript. MS and M Metzger contributed to the experiments and drafted the manuscript. JW contributed to the study concept, drafted figures, and wrote the manuscript.

\section{Acknowledgments}

These studies were supported by the Deutsche Forschungsgemeinschaft (DFG) Priority Programme (SPP 1782 to NS and JW) and by the Interdiszplinaere Zentrum fuer Klinische Forschung (IZKF) (Z-2/63 to M Meir). We thank Mohamed Hankir for critical proofreading and language editing.

Address correspondence to: Nicolas Schlegel, Department of General, Visceral, Vascular and Pediatric Surgery, University Hospital Würzburg, Oberduerrbacherstrasse 6, 97080 Wuerzburg, Germany. Phone: 49.931.201.38217; Email: schlegel_n@ukw.de.
1. Geremia A, Biancheri P, Allan P, Corazza GR, Di Sabatino A. Innate and adaptive immunity in inflammatory bowel disease. Autoimmun Rev. 2014;13(1):3-10.

2. Uhlig HH. Monogenic diseases associated with intestinal inflammation: implications for the understanding of inflammatory bowel disease. Gut. 2013;62(12):1795-1805.

3. Martini E, Krug SM, Siegmund B, Neurath MF, Becker C. Mend your fences: the epithelial barrier and its relationship with mucosal immunity in inflammatory bowel disease. Cell Mol Gastroenterol Hepatol. 2017;4(1):33-46.

4. Chang J, Leong RW, Wasinger VC, Ip M, Yang M, Phan TG. Impaired intestinal permeability contributes to ongoing bowel symptoms in patients with inflammatory bowel disease and mucosal healing. Gastroenterology. 2017;153(3):723-731.e1.

5. Farquhar MG, Palade GE. Junctional complexes in various epithelia. J Cell Biol. 1963;17:375-412.

6. Luissint AC, Parkos CA, Nusrat A. Inflammation and the intestinal barrier: leukocyte-epithelial cell interactions, cell junction remodeling, and mucosal repair. Gastroenterology. 2016;151(4):616-632.

7. Spindler V, et al. Loss of desmoglein 2 contributes to the pathogenesis of Crohn's disease. Inflamm Bowel Dis. 2015;21(10):2349-2359.

8. Gross A, et al. Desmoglein 2, but not desmocollin 2, protects intestinal epithelia from injury. Mucosal Immunol. 2018;11(6):1630-1639.

9. Spindler V, et al. Mechanisms causing loss of keratinocyte cohesion in pemphigus. J Invest Dermatol. 2018;138(1):32-37.

10. Sanders DS. Mucosal integrity and barrier function in the pathogenesis of early lesions in Crohn's disease. JClin Pathol. 2005;58(6):568-572.

11. Neunlist M, et al. Human ENS regulates the intestinal epithelial barrier permeability and a tight junction-associated protein ZO-1 via VIPergic pathways. Am J Physiol Gastrointest Liver Physiol. 2003;285(5):G1028-G1036.

12. Bush TG, et al. Fulminant jejuno-ileitis following ablation of enteric glia in adult transgenic mice. Cell. 1998;93(2):189-201.

13. Cornet A, et al. Enterocolitis induced by autoimmune targeting of enteric glial cells: a possible mechanism in Crohn's disease? Proc Natl Acad Sci U S A. 2001;98(23):13306-13311.

14. Rao M, et al. Enteric glia regulate gastrointestinal motility but are not required for maintenance of the epithelium in mice gastroenterology. Gastroenterology. 2017;153(4):1068-1081.

15 . Brun $\mathrm{P}$, et al. Toll like receptor-2 regulates production of glial-derived neurotrophic factors in murine intestinal smooth muscle cells. Mol Cell Neurosci. 2015;68:24-35.

16. Meir M, et al. Glial cell line-derived neurotrophic factor promotes barrier maturation and wound healing in intestinal epithelial cells in vitro. Am J Physiol Gastrointest Liver Physiol. 2015;309(8):G613-G624.

17. Brun P, et al. Toll-like receptor 2 regulates intestinal inflammation by controlling integrity of the enteric nervous system. Gastroenterology. 2013;145(6):1323-1333.

18. Steinkamp M, et al. Glial-derived neurotrophic factor regulates apoptosis in colonic epithelial cells. Gastroenterology. 2003;124(7):1748-1757.

19. Zhang DK, et al. Glial-derived neurotrophic factor regulates intestinal epithelial barrier function and inflammation and is therapeutic for murine colitis. J Pathol. 2010;222(2):213-222.

20. Ungewiß H, et al. Desmoglein 2 regulates the intestinal epithelial barrier via p38 mitogen-activated protein kinase. Sci Rep. 2017;7(1):6329.

21. Vielmuth F, Hartlieb E, Kugelmann D, Waschke $\mathrm{J}$, Spindler V. Atomic force microscopy identifies regions of distinct desmoglein 3 adhesive properties on living keratinocytes. Nanomedicine.
2015;11(3):511-520.

22. Majumdar D, Tiernan JP, Lobo AJ, Evans CA, Corfe BM. Keratins in colorectal epithelial function and disease. Int J Exp Pathol. 2012;93(5):305-318.

23. Durbec $P$, et al. GDNF signalling through the Ret receptor tyrosine kinase. Nature. 1996;381(6585):789-793.

24. Knowles PP, et al. Structure and chemical inhibition of the RET tyrosine kinase domain. J Biol Chem. 2006;281(44):33577-33587.

25. Gregory-Evans K, Chang F, Hodges MD, Gregory-Evans CY. Ex vivo gene therapy using intravitreal injection of GDNF-secreting mouse embryonic stem cells in a rat model of retinal degeneration. Mol Vis. 2009;15:962-973.

26. Luz M, Mohr E, Fibiger HC. GDNF-induced cerebellar toxicity: A brief review. Neurotoxicology. 2016;52:46-56.

27. Peters RJ, et al. GDNF is abundant in the adult rat gut. J Auton Nerv Syst. 1998;70(1-2):115-122.

28. von Boyen GB, Schulte N, Pflüger C, Spaniol U, Hartmann C, Steinkamp M. Distribution of enteric glia and GDNF during gut inflammation. BMC Gastroenterol. 2011;11:3.

29. Lakhan SE, Kirchgessner A. Neuroinflammation in inflammatory bowel disease. J Neuroinflammation. 2010;7:37.

30. Grubišić V, Gulbransen BD. Enteric glial activity regulates secretomotor function in the mouse colon but does not acutely affect gut permeability. J Physiol (Lond). 2017;595(11):3409-3424.

31. Löf Granström A, Amin L, Arnell H, Wester T. Increased risk of inflammatory bowel disease in a population-based cohort study of patients with Hirschsprung disease. J Pediatr Gastroenterol Nutr. 2018;66(3):398-401.

32. Nakamura H, Lim T, Puri P. Inflammatory bowel disease in patients with Hirschsprung's disease: a systematic review and meta-analysis. Pediatr 
Surg Int. 2018;34(2):149-154.

33. Reinshagen M, et al. Protective role of neurotrophins in experimental inflammation of the rat gut. Gastroenterology. 2000;119(2):368-376.

34. Langness S, Kojima M, Coimbra R, Eliceiri BP, Costantini TW. Enteric glia cells are critical to limiting the intestinal inflammatory response after injury. Am J Physiol Gastrointest Liver Physiol. 2017;312(3):G274-G282.

35. Schlegel N, Meir M, Heupel WM, Holthöfer B, Leube RE, Waschke J. Desmoglein 2-mediated adhesion is required for intestinal epithelial barrier integrity. Am J Physiol Gastrointest Liver Physiol. 2010;298(5):G774-G783.

36. Jiang K, et al. Galectin-3 regulates desmoglein-2 and intestinal epithelial intercellular adhesion. JBiol Chem. 2014;289(15):10510-10517.

37. Kamekura R, et al. Loss of the desmosomal cadherin desmoglein-2 suppresses colon cancer cell proliferation through EGFR signaling. Oncogene. 2014;33(36):4531-4536.

38. Kamekura R, et al. Inflammation-induced desmoglein-2 ectodomain shedding compromises the mucosal barrier. Mol Biol Cell. 2015;26(18):3165-3177.

39. Awad MM, Calkins H, Judge DP. Mechanisms of disease: molecular genetics of arrhythmogenic right ventricular dysplasia/cardiomyopathy. Nat Clin Pract Cardiovasc Med. 2008;5(5):258-267.

40. Schlipp A, et al. Desmoglein-2 interaction is crucial for cardiomyocyte cohesion and function. Cardiovasc Res. 2014;104(2):245-257.
41. Patel DM, Dubash AD, Kreitzer G, Green KJ. Disease mutations in desmoplakin inhibit $\mathrm{Cx} 43$ membrane targeting mediated by desmoplakin-EB1 interactions. JCell Biol. 2014;206(6):779-797.

42. Ungewiß H, et al. Dsg2 via Src-mediated transactivation shapes EGFR signaling towards cell adhesion. Cell Mol Life Sci. 2018;75(22):4251-4268.

43. Vielmuth F, et al. Keratins regulate the adhesive properties of desmosomal cadherins through signaling. J Invest Dermatol. 2018;138(1):121-131.

44. Calkins CC, et al. Desmoglein endocytosis and desmosome disassembly are coordinated responses to pemphigus autoantibodies. J Biol Chem. 2006;281(11):7623-7634.

45. Owens DW, et al. Human keratin 8 mutations that disturb filament assembly observed in inflammatory bowel disease patients. J Cell Sci. 2004;117(Pt 10):1989-1999.

46. Wang L, Srinivasan S, Theiss AL, Merlin D, Sitaraman SV. Interleukin-6 induces keratin expression in intestinal epithelial cells: potential role of keratin-8 in interleukin-6-induced barrier function alterations. J Biol Chem. 2007;282(11):8219-8227.

47. Magin TM, Vijayaraj P, Leube RE. Structural and regulatory functions of keratins. Exp Cell Res. 2007;313(10):2021-2032.

48. Fois $\mathrm{G}$, et al. Effects of keratin phosphorylation on the mechanical properties of keratin filaments in living cells. FASEB J. 2013;27(4):1322-1329.

49. Kröger C, Loschke F, Schwarz N, Windoffer R, Leube RE, Magin TM. Keratins control intercellular adhesion involving PKC- $\alpha$-mediated desmoplakin phosphorylation. JCell Biol. 2013;201(5):681-692.

50. Labun K, Montague TG, Gagnon JA, Thyme SB, Valen E. CHOPCHOP v2: a web tool for the next generation of CRISPR genome engineering. Nucleic Acids Res. 2016;44(W1):W272-W276.

51. Schweinlin M, et al. Development of an advanced primary human in vitro model of the small intestine. Tissue Eng Part C Methods. 2016;22(9):873-883.

52. Wirtz S, et al. Chemically induced mouse models of acute and chronic intestinal inflammation. Nat Protoc. 2017;12(7):1295-1309.

53. Erben U, et al. A guide to histomorphological evaluation of intestinal inflammation in mouse models. Int JClin Exp Pathol. 2014;7(8):4557-4576.

54. Flemming S, et al. Soluble VE-cadherin is involved in endothelial barrier breakdown in systemic inflammation and sepsis. Cardiovasc Res. 2015;107(1):32-44.

55. Wiegering A, et al. Reactivating p53 and inducing tumor apoptosis (RITA) enhances the response of RITA-sensitive colorectal cancer cells to chemotherapeutic agents 5 -fluorouracil and oxaliplatin. Neoplasia. 2017;19(4):301-309.

56. Riccardi C, Nicoletti I. Analysis of apoptosis by propidium iodide staining and flow cytometry. Nat Protoc. 2006;1(3):1458-1461.

57. Ebner A, et al. A new, simple method for linking of antibodies to atomic force microscopy tips. Bioconjug Chem. 2007;18(4):1176-1184. 